\title{
Boundary-Layer Stability Analysis of the Mean Flows Obtained Using Unstructured Grids
}

\author{
Wei Liao* \\ National Institute of Aerospace, Hampton, VA 23666, USA \\ Mujeeb R. Malik, ${ }^{\dagger}$ Elizabeth M. Lee-Rausch,${ }^{\ddagger}$ Fei Li,${ }^{\S}$ Eric J. Nielsen, ${ }^{\natural}$ \\ Pieter G. Buning, "Chau-Lyan Chang, ${ }^{* *}$ and Meelan Choudhari ${ }^{\dagger \dagger}$ \\ Computational AeroSciences Branch, NASA Langley Research Center, Hampton, VA 23681, USA
}

\begin{abstract}
Boundary-layer stability analyses of mean flows extracted from unstructured-grid NavierStokes solutions have been performed. A procedure has been developed to extract mean flow profiles from the FUN3D unstructured-grid solutions. Extensive code-to-code validations have been performed by comparing the extracted mean flows as well as the corresponding stability characteristics to the predictions based on structured-grid solutions. Comparisons are made on a range of problems from a simple flat plate to a full aircraft configuration-a modified Gulfstream-III with a natural laminar flow glove. The future aim of the project is to extend the adjoint-based design capability in FUN3D to include natural laminar flow and laminar flow control by integrating it with boundary-layer stability analysis codes, such as LASTRAC.
\end{abstract}

\section{Nomenclature}

$C=$ wing/airfoil chord length

$C_{p}=$ Pressure coefficient

$f=$ disturbance frequency (HZ)

$H=$ flight altitude (feet)

$m=$ meter

$M a=$ free stream Mach number

$N=\mathrm{N}$ factors

$r 1=$ geometric stretching rate used in VGRID

$r 2=$ the second stretching rate used to tune cell expansion rates in VGRID.

$R_{C F}=\frac{\left|U_{C F \max }\right| \delta_{01}}{\nu_{e}}$, crossflow Reynolds number

$R e=$ free stream Reynolds number

$R e_{y}=\frac{U_{e} x}{\nu_{e}}$, Reynolds number based on the boundary layer edge velocity

$s=$ second

$U_{\infty}=$ free stream velocity

$U_{C F \max }=$ maximum crossflow velocity

$U_{e}=$ boundary layer edge velocity

$X, x=$ chordwise coordinate

$Y, y=$ spanwise coordinate

*Research Scientist, wei.liao@nasa.gov or wliao@nianet.org. Senior Member, AIAA.

†Senior Aerodynamicist, m.r.malik@nasa.gov. Fellow, AIAA.

${ }^{\ddagger}$ Aerospace Technologist, e.lee-rausch@nasa.gov. Senior Member, AIAA.

$\S$ Aerospace Technologist, fei.li@nasa.gov.

ฯAerospace Technologist, e.j.nielsen@larc.nasa.gov. Senior Member, AIAA.

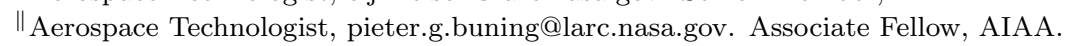

**Aerospace Technologist, chau-lyan.chang@nasa.gov. Senior Member, AIAA.

${ }^{\dagger \dagger}$ Aerospace Technologist, m.m.choudhari@nasa.gov. Associate Fellow, AIAA. 
$Z, z=$ vertical coordinates

$\delta_{99}=$ boundary layer thickness (distance from surface to $99 \%$ of free-stream velocity)

$\delta_{01}=$ distance from wall where $U_{C F}$ reduces to $0.1\left|U_{C F \max }\right|$

$\Delta_{n}=$ normal grid spacing height for the $\mathrm{n}$-th layer

$\nu=$ kinematic viscosity

$\nu_{e}=$ boundary layer edge kinematic viscosity

$\sigma=$ growth rate of disturbance

$\alpha=$ angle of attack

$\lambda=$ spanwise wavelength (millimeter)

\section{Introduction}

The reduction of aerodynamic drag on flight vehicles is becoming an increasingly more crucial issue due to the stringent energy efficiency requirements of today. The skin friction drag is an important drag component and can make up to $50 \%$ of the overall vehicle drag. Therefore, a natural laminar flow (NLF) over an aircraft configuration is desirable because of the lower skin friction associated with it, as compared with turbulent flows. In situations in which natural laminar flow is unattainable, laminar flow control (LFC) may be employed to maximize the region of laminar flow. Affordable analysis methods that adequately account for transition from laminar to turbulent flows are critical for a viable laminar flow technology and should be a part of the design process for NFL and LFC applications. The direct numerical simulations (DNS) approach to resolve the boundary-layer instability scales in the laminar-turbulent transition process is prohibitively expensive because of the very fine computational grid required. LASTRAC ${ }^{1,2}$ is a stability analysis code which is widely used to predict transition in practical applications.

In recent years, adjoint based design optimization methodology has been developed and implemented in several large-scale computational fluid dynamics (CFD) codes. FUN3D, ${ }^{3-8}$ an unstructured CFD code developed at NASA Langley, provides extensive adjoint-based capabilities for design optimization for many design parameters. The ultimate long-term goal of the research conducted by the authors is to develop an adjoint formulation for LASTRAC and integrate it with the adjoined-based capabilities of FUN3D to enable design optimization that directly maximizes the laminar-flow regions. In a short term, it is planned to analyze aerodynamic flows with various stability analysis codes, identify flow characteristics critical for delaying transition, and include these characteristics in the objective function optimized by the existing adjoint-based method.

Central to the latter approach is the extraction of accurate boundary-layer mean flows on which stability analysis is based. The boundary-layer mean flows required by stability analysis codes can be obtained by, among a variety of methods, direct computations using boundary-layer codes or extractions from NavierStokes solutions. Simple boundary-layer methods for 2D, quasi-3D or conical configurations fail when the underlying flow takes on strong three-dimensional characteristics, for which the Navier-Stokes approach may be called for. A possible alternative to the Navier-Stokes approach is the 3D boundary-layer method, which will not be discussed in this paper.

As a first step, a procedure has been developed to extract "stability quality" mean flows from flow solutions on unstructured tetrahedral and mixed-element grids, for which extensive validations are performed against mean flows extracted from structured-grid solutions ranging from a simple flat plate flow to an infinite swept wing flow to a realistic flow configuration of a composite glove on the wing of a Gulfstream-III (G-3) aircraft.

A modified Gulfstream-III aircraft fitted with a composite glove on one of its wings has been selected by NASA's Environmentally Responsible Aviation (ERA) Project to evaluate a certain laminar flow control concept for potential applications on transport aircraft. ${ }^{9,10}$ A new gloved wing was designed with NLF techniques through collaborative research between Texas A \& M University and NASA Dryden Flight Research Centers. ${ }^{11}$ The glove as installed on the G-3 aircraft wing is shown in Fig. 1. The G-3 glove is thus a perfect test case for demonstrating the capability of the extraction procedure for realistic applications. FUN3D has been used for 3D CFD simulations of the G-3 aircraft. ${ }^{10}$ Stability analysis on the mean flows produced by boundary-layer codes, which used $C_{p}$ obtained from the FUN3D solution as the boundary-layer edge condition, has also been conducted to provide some insight into the glove design. ${ }^{10}$ In the current study, the stability analysis results based on the mean flows directly extracted from FUN3D solutions are compared 

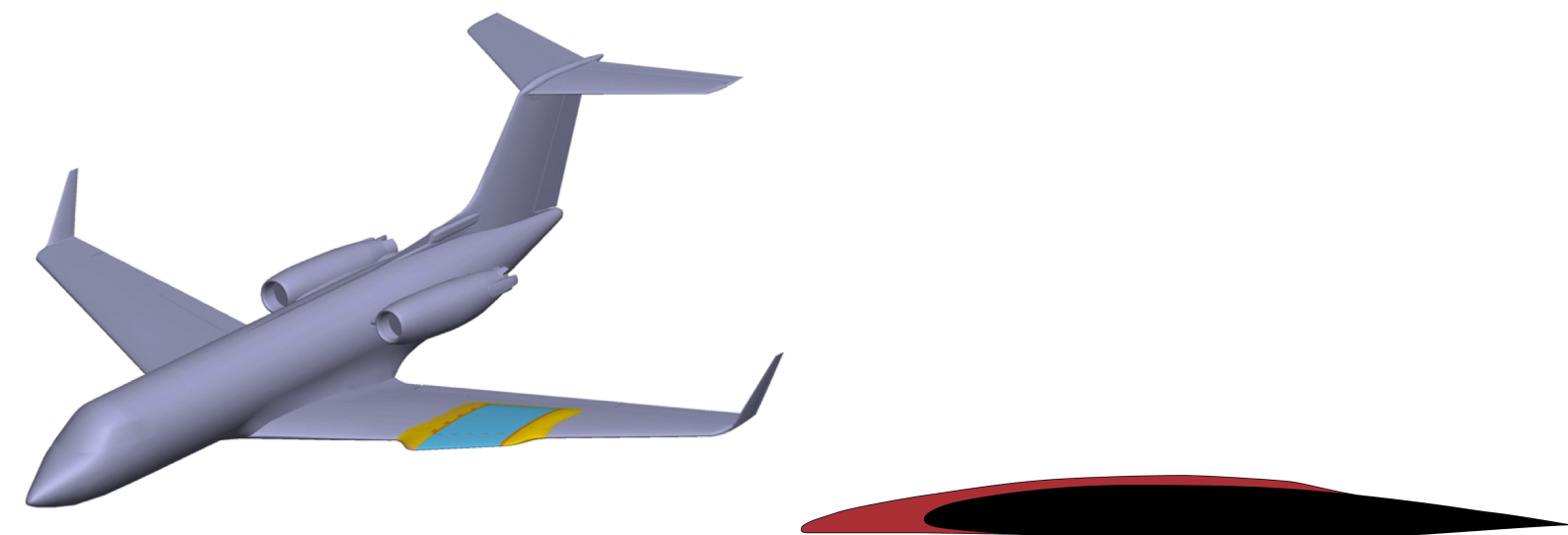

Figure 1. G-3 aircraft with a composite glove (left) and cross section of the wing-glove (right).

to those based on other well-established codes, such as CFL3D ${ }^{12,13}$ and OVERFLOW. ${ }^{14,15}$ CFL3D has been extensively used for a number of laminar flow applications associated with stability analysis ${ }^{16-18}$ while OVERFLOW has not been reported for this purpose. Due to its capability of handling complex aerodynamic configurations, OVERFLOW is used here to perform computations for the full G-3 aircraft case and the results are compared with FUN3D results for cross validations.

It should be noted that, although it is tested on mean flows in which crossflow instability modes dominate, the use of the extraction procedure is not limited to just these type of flows.

The rest of the paper is structured as follows. An overview of the computational tools is given in Section II, followed by the presentation of results and discussions in Section III. Finally, conclusions are drawn in Section IV.

\section{Computational Tools}

A general stability analysis procedure involves the following steps. First, flow solutions are obtained by CFD solvers. Then mean boundary-layer flow profiles can be obtained from the flow solutions by either of two means: 1) with boundary layer well resolved by a Navier-Stokes solver, mean flow profiles may be extracted directly from computed laminar solutions; or 2) the surface pressure and temperature from the CFD solutions are imposed as the edge conditions for boundary layer codes to generate mean flow quantities. Finally, with the obtained boundary layer mean flows as a basis, stability analysis codes can be used to predict the linear and/or nonlinear development of instability modes. In this work, different tools or approaches may be used for cross-validation purposes at each step. The tools or approaches used here are briefly introduced below.

\section{A. Numerical Tools for Flow Computations}

\section{FUN3D - Unstructured-Grid Flow Solver}

FUN3D ${ }^{3-8}$ is a suite of computational fluid dynamics (CFD) codes, developed at NASA Langley, based on fully unstructured grids for flow analysis, adjoint-based design optimization, error estimation, and objective based adaptive mesh refinement. FUN3D solves the Reynolds-Averaged Navier-Stokes (RANS) equations using a node-centered second-order upwind implicit scheme. FUN3D can solve the equations on mixed element grids, including tetrahedra, pyramids, prisms, and hexahedra. At control volume interfaces, the inviscid fluxes are computed using an approximate Riemann solver based on the values on either side of the interface. Several convective flux schemes are available in FUN3D. The most common scheme for subsonic and transonic flows is Roe's flux difference splitting, ${ }^{19}$ which is used in the current study.

FUN3D has an adjoint-based optimization module providing a very efficient design capability for complex aerodynamic configurations. The current study is the first step to couple the flow analysis and adjoint design capability of FUN3D with the NASA stability and transition prediction tool, LASTRAC, for NLF or LFC wing design. 


\section{VGRID - Unstructured Grid Generation}

The unstructured-grid tool VGRID, ${ }^{20}$ based on the Advancing-Front (AFM) and the Advancing-Layers (ALM) methods, is employed to generate tetrahedral grids in the current study. The generation of a viscous grid is divided into three main steps: (1) generation of triangular surface grid by the ALM and/or AFM, (2) generation of thin tetrahedral cells in the boundary layer by the ALM, and (3) generation of regular (inviscid) tetrahedral grid outside the boundary layer by the AFM. VGRID uses the following formula to establish the normal spacing for the $\mathrm{n}$-th layer in the advancing layers, ${ }^{20}$

$$
\Delta_{n}=\Delta_{1}\left[1+r_{1}\left(1+r_{2}\right)^{n-1}\right]^{n-1}
$$

where $\Delta_{1}$ is the normal spacing of the first layer, $r_{1}$ is a geometric stretching rate, and $r_{2}$ is the stretching rate which slows down the rate of cells opening up and then accelerate the expansion rate. Variable stretching ratios are allowed to use a set of smaller stretching ratios in the area of interest while using larger ratios elsewhere.

\section{CFL3D and OVERFLOW - Structured-Grid Flow Solvers}

CFL3D $^{12,13}$ is a well-known three-dimensional structured-grid flow solver developed from 1980's at NASA Langley. CFL3D solves the time-dependent conservation law form of the RANS equations using a semidiscrete finite-volume approach with upwind-biasing of the convective and pressure terms and central differencing of the shear stress and heat transfer terms. In the current study, the Roe flux scheme ${ }^{19}$ is chosen for flow analysis. One of the code's many strengths is the diversity of available turbulence models. CFL3D can handle one-to-one multi-block grids, patched grids, and overset grids as well.

OVERFLOW ${ }^{14,15}$ is a Navier-Stokes flow solver developed by NASA for structured grids, based on single block grids or overset (structured) grid systems. When solving the flow equations, the convective term discretization options include central differencing with Jameson's artificial dissipation scheme ${ }^{21}$ and Roe flux scheme. ${ }^{19}$ Grid sequencing and multigrid are implemented to accelerate convergence coupled with overset grid techniques. OVERFLOW is used to compute the full G3 aircraft case with the Roe's scheme in the grid block over the wing and the artificial dissipation scheme in the other blocks for better computational efficiency.

CFL3D and OVERFLOW are used in the present study for validation purposes.

\section{B. Stability Analysis Tools}

Based on the boundary-layer mean flow profiles obtained, LASTRAC ${ }^{1,2}$ can be utilized to predict linear and/or nonlinear development of instability modes. The analysis can be conducted for both crossflow and Tollmien-Schlichting (TS) waves with three approaches: (i) Linear (Parallel) Stability Theory (LST); (ii) linear Parabolized Stability Equations (PSE); and (iii) Non-linear Parabolized Stability Equations (NPSE). Stability calculations and N-factor correlations can be performed for both $2 \mathrm{D} /$ axisymmetric $^{1}$ and general $3 \mathrm{D}$ configurations. $^{2}$

The 3D transition analysis module within LASTRAC is based on boundary layer profiles at each point of a structured grid surface mesh. A mean flow is usually extracted directly from Navier-Stokes solutions and fed to LASTRAC for 3D analysis. When dealing with 3D boundary layers, LASTRAC can solve for disturbance evolution by using either surface-marching approach or local line-marching approach. For practical applications, the local line-marching method is usually used for transition correlations due to its numerical efficiency. In the current study, we use local streamline-marching for 3D stability analysis due to its simplicity and efficiency. A 2D stability analysis is usually conducted based on the mean flow profiles obtained by $2 \mathrm{D}$ or quasi-3D boundary layer codes.

eMalik, ${ }^{22}$ based on linear stability theory for stability analysis of $2 \mathrm{D}$ or axisymmetric compressible wallbounded flows, is also used for validation purposes when necessary.

\section{Boundary Layer Codes for Boundary Layer Profiles}

With the surface pressure and temperature from CFD computations or experiments as edge conditions for boundary layer codes, mean flow profiles can be generated for stability analysis. Here two boundary layer codes are used for this purpose. WINGBL2 is a spectral boundary-layer solver developed by Pruett ${ }^{23}$ for an 
infinite swept wing. Due to its infinite span approximation, only mean flow profiles with no spanwise variation in quantities can be produced. BLSTA is an efficient finite difference boundary-layer solver with second-order accuracy, which is able to solve 2D, axisymmetric or quasi-3D laminar boundary layer equations. ${ }^{24}$ BLSTA can be used for infinite swept wings, swept-back or -forward tapered wings with a spanwise conical flow assumption, and even swept-back delta (low aspect ratio) wings with a streamwise conical flow assumption.

\section{Direct Extraction of Boundary Layer Profiles from Navier-Stokes Solutions}

Besides resorting to boundary layer codes, one can extract boundary-layer profiles directly from laminar solutions computed by a Navier-Stokes solver. Boundary layer mean flow profiles may be directly taken from the flow fields obtained by structured-grid flow solvers like CFL3D and OVERFLOW. However, since FUN3D is an unstructured flow solver, it is not straightforward to obtain detailed boundary layer profiles from its solutions directly. As a result, an extraction procedure has been developed to construct an appropriate boundary layer grid which is orthogonal and clustered near the wall boundary to capture instability modes.

In the developed extraction procedure, the leading edge of the wing, from which the stability grid starts to be constructed, is automatically detected by a binary search iterative procedure. The detected leading edge forms one edge of a 2D Cartesian grid. By specifying the domain extent in the streamwise and spanwise direction, users can thus define this Cartesian grid completely. Then FUN3D projects the 2D grid onto the wing surfaces to obtain seed points. Profile points are then distributed along the wall normal vectors computed at each seed point according to users' input. The extent in the normal direction is determined by the boundary layer thickness which can be estimated by ${ }^{25}$

$$
\delta_{99}(x)=5 \sqrt{\frac{\nu x}{U_{\infty}}}
$$

where $\nu$ is the kinematic viscosity, $x$ is the streamwise distance downstream from the start of the boundary layer, and $U_{\infty}$ is the free stream velocity. With the boundary layer thickness estimated by Eq. (2), the outer edge of the stability grid can be determined accordingly. The constant in Eq. (2) can be adjusted to account for pressure gradient effects. A stretching function is implemented to cluster the stability grid at the areas near the leading edge and the wall surface. A single-plane structured grid is established at each spanwise location. Users can define the number of spanwise locations based on resolution requirements. Fig. 2 illustrates a sample stability grid established by the current projection procedure.

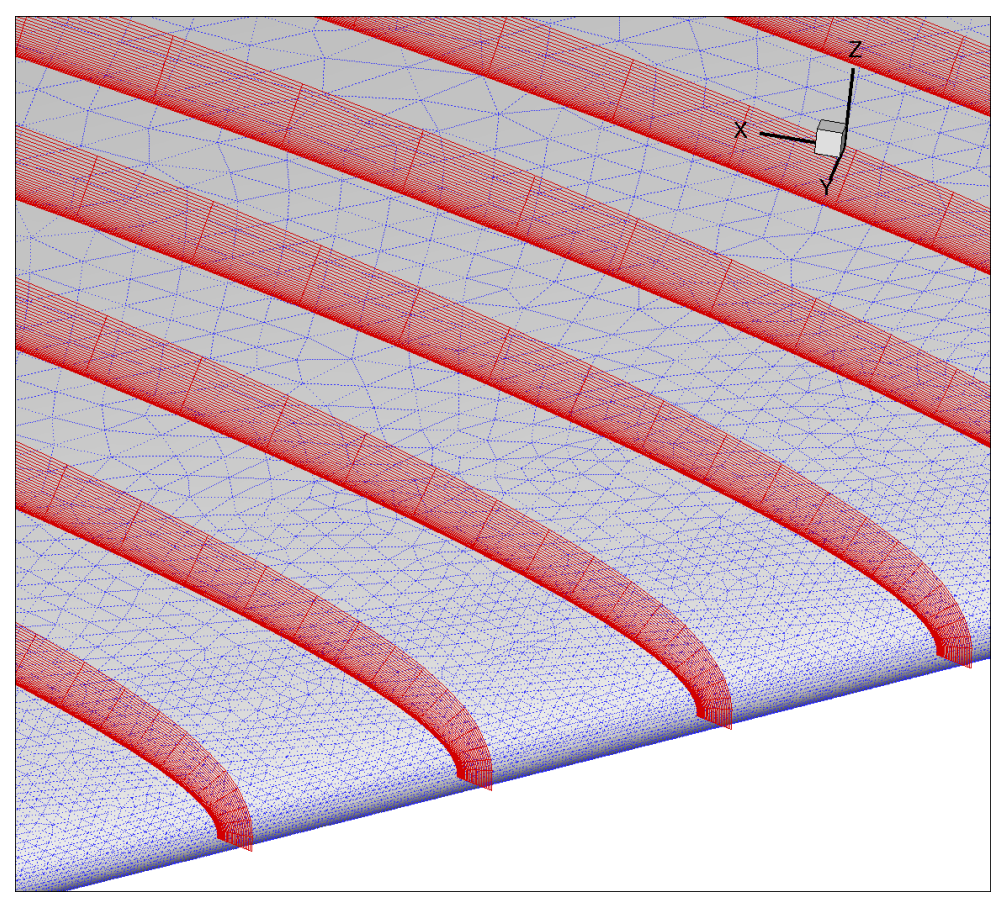

Figure 2. A sample stability grid established by the developed extraction procedure. 
FUN3D identifies the unstructured grid element containing each profile point on the stability grid. The flow variables on the newly constructed grid are then interpolated from their values at the surrounding grid points in the element. FUN3D can solve the equations on mixed element grids, including tetrahedra, pyramids, prisms, and hexahedra. However, interpolation is always based on tetrahedral interpolation no matter what kind of elements are used. To interpolate at a particular point, the first step is to determine which mesh element the point is in. If this element is not tetrahedral, this element will be temporarily subdivided into tetrahedra. A hexahedron, prism and pyramid can be subdivided into six, three and two tetrahedra, respectively. Once the tetrahedron containing the interpolation point is identified through a searching procedure, quantities are computed with first-order accuracy as a weighted sum of the values at the vertices of that tetrahedron based on the barycentric coordinates of the interpolation point.

The above extraction process has been parallelized and integrated to FUN3D. With this procedure, the stability grid is able to provide Navier-Stokes solution based mean flow profiles for boundary layer stability analysis immediately after a CFD computation is done. The profiles extracted from unstructured data are output as multi-block PLOT3D grid and solution files.

\section{E. Laminar Region Specification}

Mean flows for stability analysis must be based on laminar flow solutions. Therefore, whenever we are concerned with the instability of the laminar boundary layer in selected regions of the wing, the laminar flow must be imposed on the corresponding zones while turbulence models are used for all the other regions. The CFD solvers used here have recently incorporated transition-based turbulence models such as that described in Ref. ${ }^{13}$ However, laminar flow regions are manually specified in this study.

A laminar flow specification approach has been incorporated into FUN3D, by which multiple laminar flow regions in the flow field can be manually specified based on the flow physics and numerical requirements. In this approach, arbitrary line sections are introduced to define different laminar zones and multiple transition locations in flow fields. As a result, laminar and turbulent regions are defined upstream and downstream of the transition locations. The approach implemented in FUN3D, CFL3D, and OVERFLOW to specify transition locations is based on the idea of turning off the turbulent production terms in the "laminar" regions of the grid. ${ }^{26}$ The flow usually remains laminar inside the specified regions as a result. Full turbulence model equations are used everywhere although their turbulence production terms are turned off in the specified laminar regions. Consequently, turning off the production term may not prevent turbulence from convecting or diffusing into laminar regions near the transition boundaries. So the specified laminar regions should usually be larger than the areas that we are interested in. The values of turbulence viscosity should be checked to ensure laminar flows in the areas of interest.

Since both CFL3D and OVERFLOW are structured grid flow solvers, the laminar region specification in them is based on $(i, j, k)$ grid point indexes in a grid block instead of $(x, y, z)$ coordinates used in FUN3D. As a result, when these codes are used to compute the same case, the laminar flow regions specified may have some slight difference. Note that in the current study, the Spalart-Allmaras ${ }^{27}$ model is always chosen for turbulence computations although a variety of turbulence models are available in all three codes.

\section{Results and Discussions}

This section presents the results of computations aimed at validating the mean flow extraction procedure. The first two cases involve relatively simple flow configurations of a flat plate and an infinite swept wing. Then the more realistic case of a simplified G-3 wing glove assembly is examined, in which the fuselage, engine, and winglet are removed. Finally, computations of the full G-3 aircraft are carried out.

\section{A. Flat Plate}

The first case to be examined is the flow over a semi-infinite flat plate for which a similarity solution exists. The free stream Mach number and unit Reynolds number are chosen to be 0.5 and $1 \times 10^{6}$ per meter, respectively. FUN3D is used to compute the laminar flow field in a computational domain that extends to $1 \mathrm{~m}$ upstream and $3 \mathrm{~m}$ downstream of the flat-plate leading edge, respectively, with a spanwise width of $1 \mathrm{~m}$ and an upper boundary fixed at $0.06 \mathrm{~m}$ (approximately 6 times the boundary layer thickness at the end of the computational domain). A very fine unstructured grid with pure tetrahedron cells is generated by VGRID ${ }^{20}$ to resolve the boundary layer. The grid spacing is determined by Eq. (1) with $r_{1}=0.028$, 
$r_{2}=0$, and $\Delta_{1}=6 \times 10^{-6} \mathrm{~m}$. Figure 3 shows the grid distribution on the wall surface $(\mathrm{X}-\mathrm{Y})$ and at the midspan cut normal to the wall (X-Z). For validation purposes, CFL3D computations are also conducted on the structured grids with two resolutions, $257 \times 193$ and $513 \times 385$.
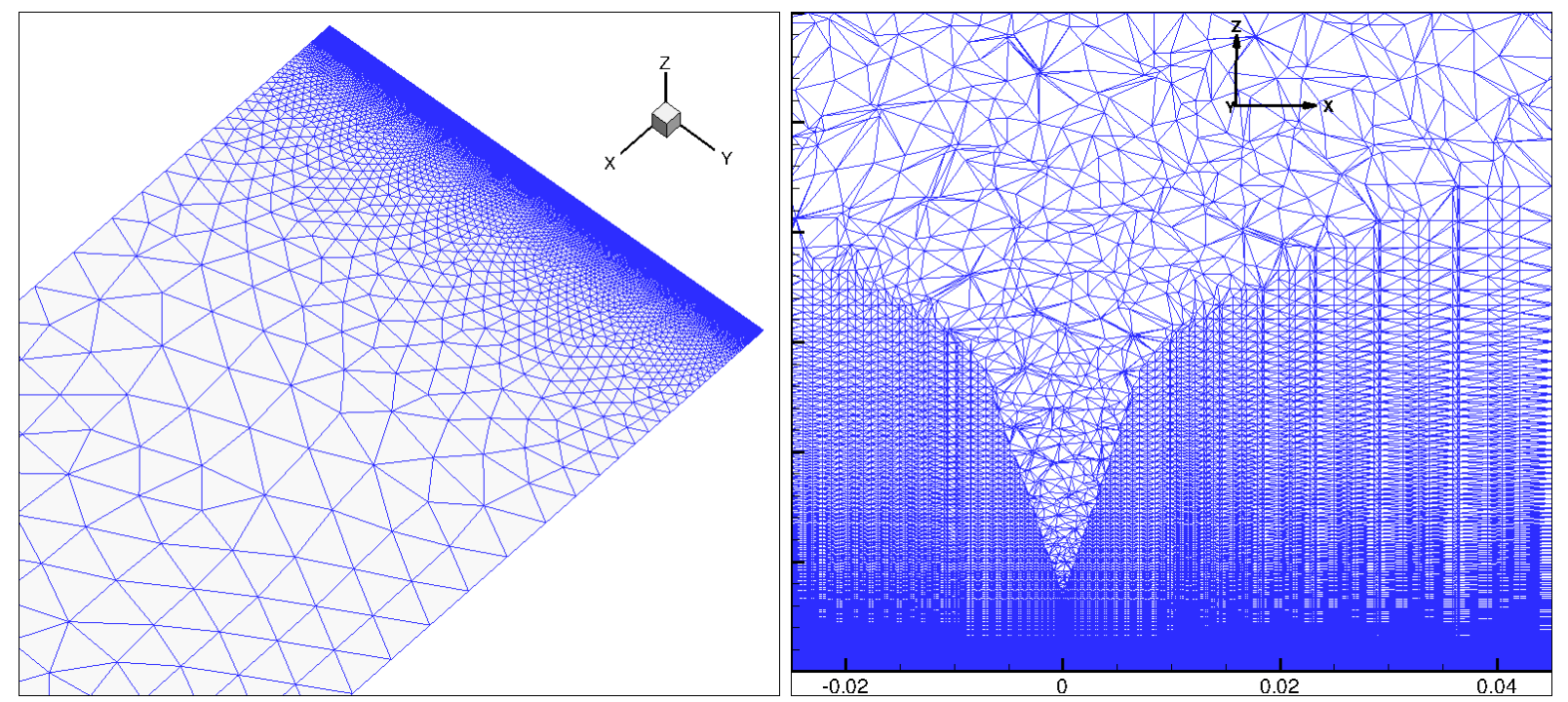

Figure 3. Unstructured grid in $\mathrm{X}-\mathrm{Y}$ plane (left) and $\mathrm{X}-\mathrm{Z}$ plane (right) for the flat plate.

The extraction procedure is employed to obtain boundary-layer profiles from the FUN3D solution. Comparisons of the streamwise velocity profiles with the CFL3D solution and the Blasius similarity solution are shown in Fig. 4 up to the second derivative. The agreements are excellent.
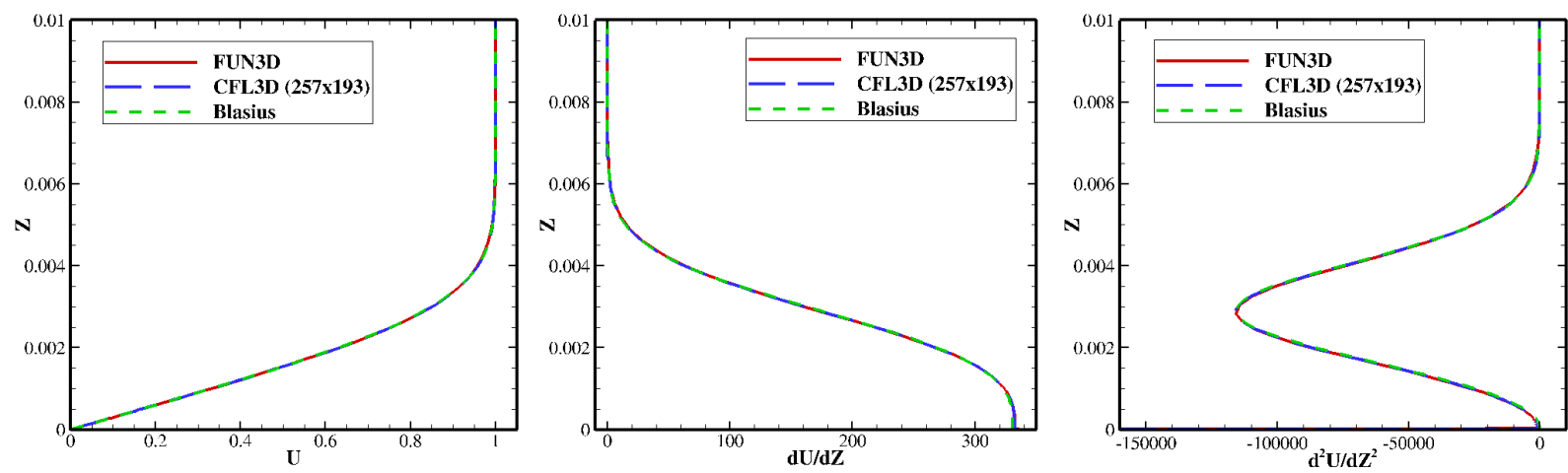

Figure 4. The profiles of the velocity and its 1st-order and 2nd-order gradients over the flat plate.

Stability computations of TS instability waves are performed next by LASTRAC based on FUN3D and CFL3D solutions and by $\mathrm{eMalik}^{22}$ based on the similarity solution. The instability growth rates at two spatial locations, $x=0.945577 \mathrm{~m}$ and $x=1.96839 \mathrm{~m}$, are shown in Fig. 5 . The results from different computations are in good agreements. Numerical tests also reveal that, while the residual of the numerical solution needs to converge by only 3 to 4 orders of magnitude for the computation of aerodynamic forces to engineering accuracy, it must converge by 6 to 7 orders of magnitude if the solution is intended for stability analysis.

\section{B. Infinite Swept Wing}

The second test case is a wing of $34.58^{\circ}$ sweep angle, at $2.3^{\circ}$ of angle of attack, with an infinite span. Its cross section has the same shape as that of the G-3 wing-glove shown in the right plot of Fig. 1 . The free stream Mach number and the chord Reynolds number are, respectively, 0.75 and $22 \times 10^{6}$. Both CFL3D and FUN3D are used for computations. The structured grid for the CFL3D computation has a grid size of $737 \times 5 \times 257$ 

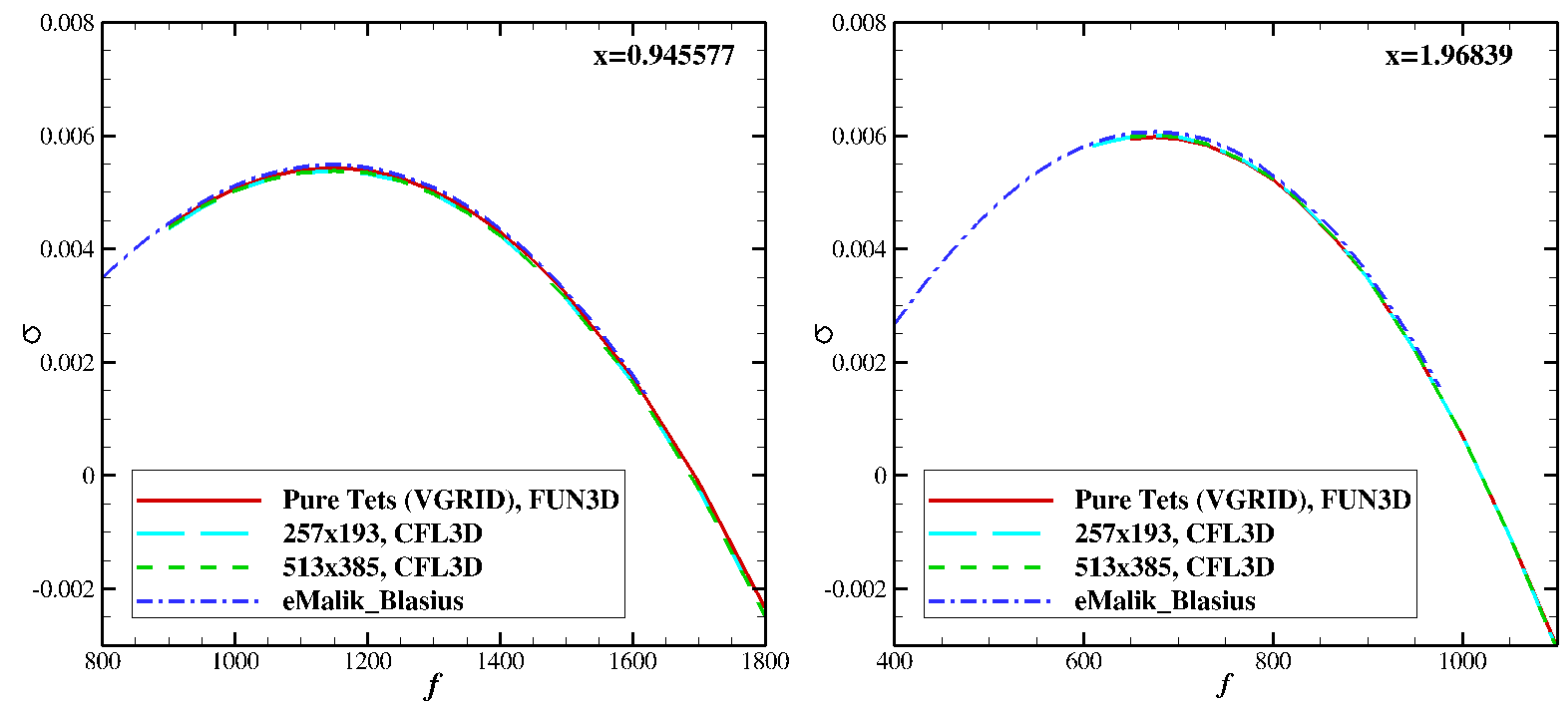

Figure 5. Growth rate vs. disturbance frequency at two spatial locations over flat plate.

in streamwise, spanwise and wall-normal directions, respectively, with the outer boundary extending to distances of approximately 50 times the chord length away from the wing surface in all directions. This grid is then converted to an unstructured grid for FUN3D computations. The extraction procedure is used to obtain mean flows from the solutions based on tetrahedron cells. For both computations, the periodic boundary conditions are used in the spanwise direction.

The surface pressure coefficient, $C_{p}$, is extracted from the FUN3D solution and is compared in Fig. 6(a) with that from the CFL3D solution. The agreement is excellent. The extracted surface pressure coefficient distribution is then used for the generation of a mean flow by the WINGBL2 boundary-layer code. The mean flows respectively extracted from the FUN3D solution and generated by WINGBL2 boundary-layer solution are used for linear stability analysis by LASTRAC. Figure 6(b) shows the N-factors for stationary crossflow instability modes of 4, 7, 10 and $13 \mathrm{~mm}$ in spanwise wavelength, respectively. The agreement between $\mathrm{N}$-factors based on the mean flows of different origins is quite good.
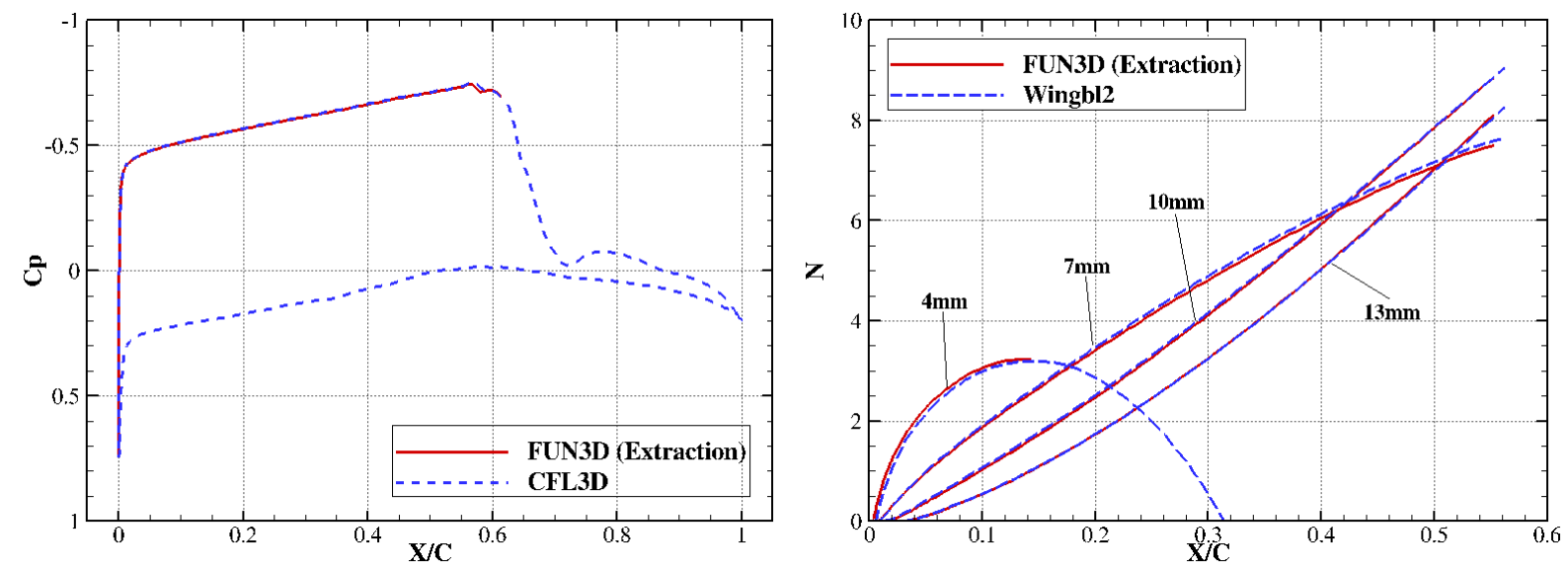

Figure 6. (a) $C_{p}$ distributions from FUN3D through the extraction procedure and CFL3D (left); (b) Stationary crossflow LST N-factors based on the mean flows from FUN3D and WINGBL2.

\section{Gloved-Wing Only}

The validation tests carried out so far in subsections A and B above show that the extraction procedure works satisfactorily, at least for simple flow configurations. In this subsection, computations are performed 
for a simplified G-3 wing-glove assembly with the fuselage, engine and winglet removed from the full aircraft for computational expediency.

For this configuration the glove leading-edge sweep angle is slightly larger than that of the wing itself, i.e. $34.58^{\circ}$ and $31.7^{\circ}$ for the former and the latter, respectively. The wing trailing-edge sweep angle is $13.62^{\circ}$. The freestream Mach number and the chord Reynolds number are, respectively, 0.75 and $22 \times 10^{6}$ with the ambient pressure and temperature chosen to be those at an altitude of 38,400 feet in the standard atmosphere. The wing-glove assembly has an angle of attack of 3.4 degrees.

Two unstructured grids are generated by VGRID ${ }^{20}$ with mixed elements having approximately 68.9 and 46.6 million nodes, respectively, for FUN3D viscous flow computations. The mixed-element grids consist of tetrahedra, pyramid, and prisms where the prisms are generated by recombining the tetrahedral grid in the advancing layers. The finer grid consists of 23.8 million tetrahedra, 128.4 million prisms, and 1.2 million pyramids while the coarser grid has around 24.7 million tetrahedra, 83.7 million prisms, and 0.96 million pyramids. The grid spacing for both meshes is determined by Eq. (1) with $\Delta_{1}=1.4 \times 10^{-4}$ to ensure that $y^{+}<1$ everywhere. Since uniform stretching ratio is used for satisfactory grid quality, the grid sizes become relatively large as a result. The stretching ratios are $r_{1}=0.0125$ and $r_{2}=0.01$ for the finer grid, and $r_{1}=0.03$ and $r_{2}=0.01$ for the coarser grid. The number of nodes inside the boundary layer at any fixed location is approximately 80 and 50 , respectively, for the two grids.

A multi-block structured grid with a total number of 112 million points is generated by GRIDGEN for CFL3D simulations. There are approximately 110 points inside the boundary layers at any location in the glove region. Figure 7 shows both the unstructured and structured surface meshes of the wing-glove assembly.

For FUN3D computations the residual for the mass conservation is driven to decrease by 7 orders of magnitude and for the CFL3D computations it is decreased by 6 orders of magnitude. Consistency in the solutions can be seen in surface pressure coefficient contours shown in Figure 8(a) and (b).
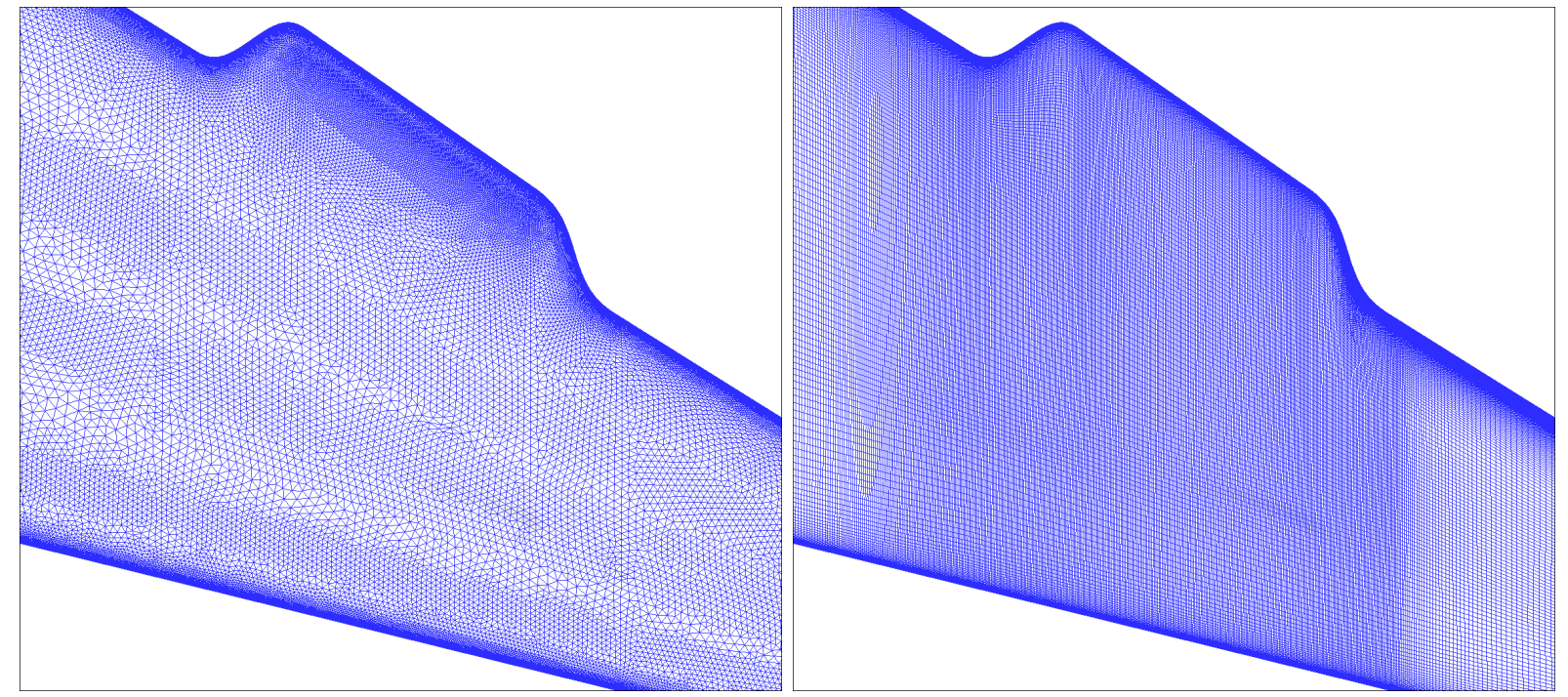

Figure 7. Unstructured (left) and structured (right) grid used for FUN3D and CFL3D, respectively.

Since the current case is fully 3D, the 3D capability of LASTRAC is utilized for stability analysis. Two sets of mean flows over the glove region are respectively extracted from the coarser and finer FUN3D unstructured grid solutions, and a third set is directly obtained from the CFL3D structured grid solution. For both FUN3D mean flows, 61 stations are extracted over the width of the glove. Linear stability analysis is performed on each of the mean flows for stationary crossflow instability modes with spanwise wavelengths of 5, 7, 9 and $11 \mathrm{~mm}$, respectively. The N-factors based on the three mean flows along a streamline that starts from a point at the mid section of the glove near the leading edge are shown on the left of Fig. 9 with the the streamline itself shown on the right. The similarity of the three streamlines derived from three different solutions is observed. The general agreements are good among the $\mathrm{N}$-factor curves. The discernible, but small differences are acceptable given that the $\mathrm{N}$-factor is quite sensitive to perturbations to the mean flow. A useful conclusion can be drawn from this exercise that, even with only 50 points in the boundary-layer resolution, the stability analysis yields good results. 

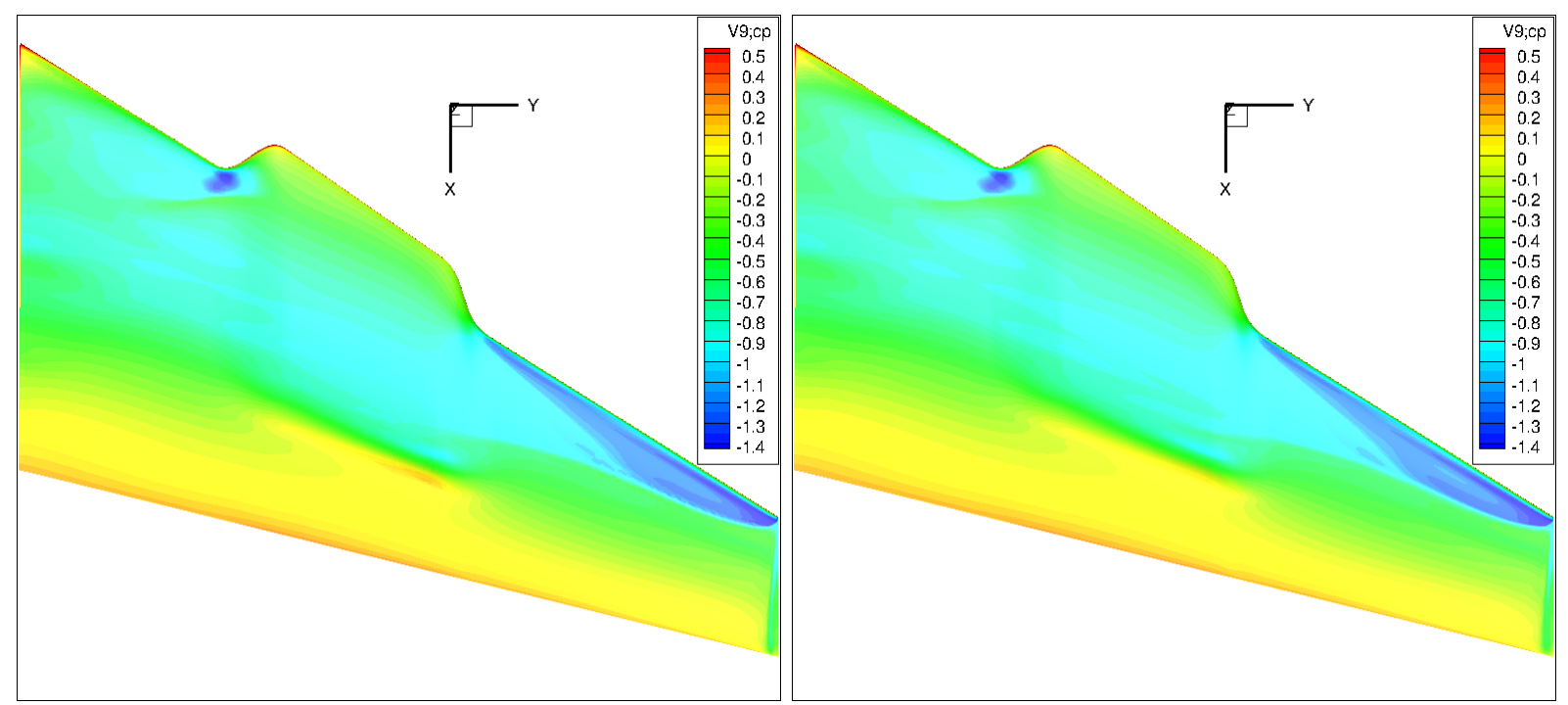

Figure 8. $C_{p}$ contours for FUN3D (left) and CFL3D (right).

In addition to streamwise and wall-normal resolutions, the spanwise resolution used to extract the mean flow could also affect the accuracy of stability analysis. Therefore, a new mean flow is extracted from the finer FUN3D solution with an increased spanwise resolution, i.e. the number of spanwise stations over the width of the glove is increased from 61 to 91 . N-factors for the same stationary crossflow instability modes are plotted in Fig. 10 based on the two mean flows with different spanwise resolutions. The agreement is good, showing that 61 stations in the spanwise direction are sufficient to yield accurate stability results for the instability modes computed herein.
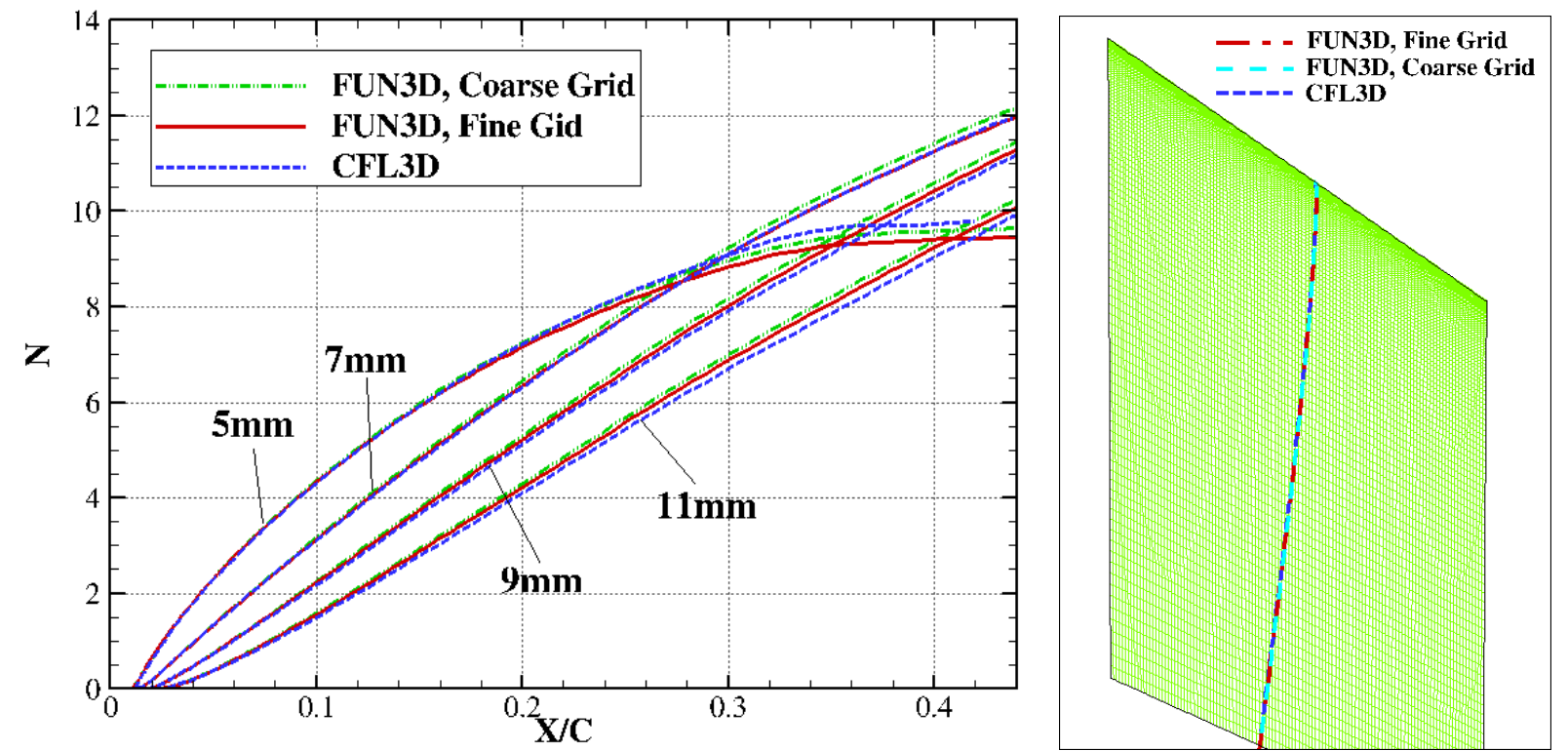

Figure 9. 3D LST N-factors for four stationary crossflow modes, 5, 7, 9, and $11 \mathrm{~mm}$, based on the mean flow profiles obtained from FUN3D and CFL3D solutions (left) and the corresponding streamlines along which the stability analysis is conducted (right). 


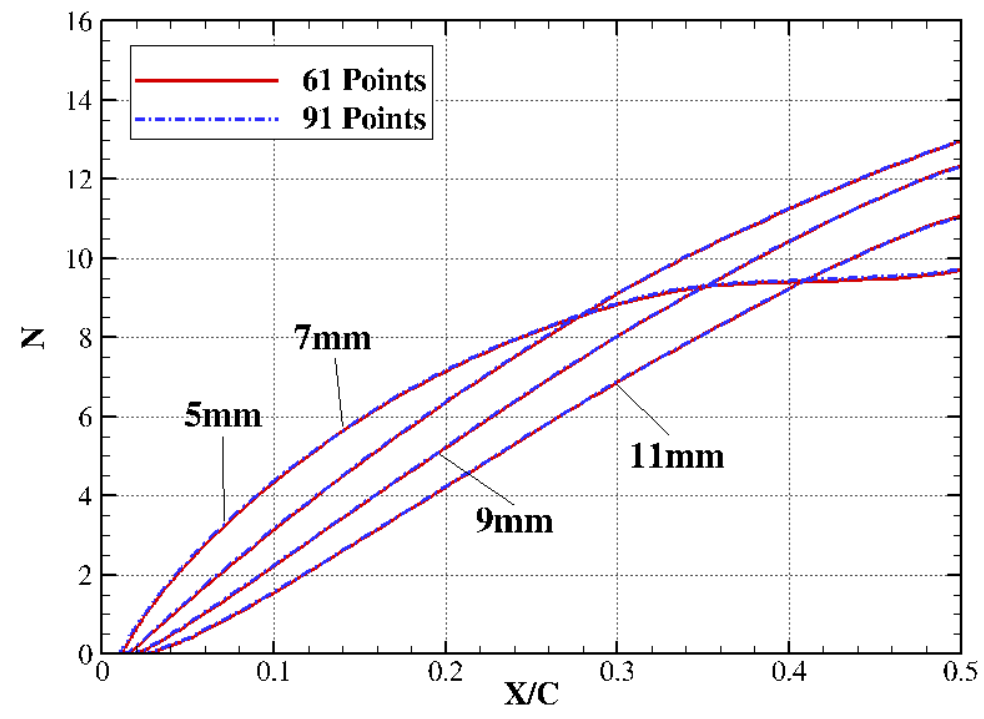

Figure 10. Comparison of the 3D LST N-factors for four stationary crossflow modes, 5, 7, 9, and $11 \mathrm{~mm}$, by using 61 and 91 spanwise stations in the extraction procedure

\section{Full G-3 Aircraft with the Gloved Wing}

In this section, computations over the full G-3 aircraft configuration including the fuselage, engine and winglet are performed. In this case, only one-half of the aircraft is gridded as flow symmetry is assumed along the centerline of the fuselage. Since the region of particular interest for this study is the mid-span region of the wing, the tail is not simulated and some minor details of the engine nacelle geometry are ignored. The free stream flow conditions are exactly the same as those for the wing-glove only case. Powered engine conditions as provided by Ref. ${ }^{28}$ are used for computation of the aircraft flow field. A mixed-element unstructured grid is generated by VGRID ${ }^{20}$ with a total of 25.7 million nodes. The near-wall mesh consists of 38.8 million prismatic elements, the far-field mesh consists of 34.5 million tetrahedron elements, and 0.48 million pyramids work as transitional elements. The unstructured grid generated for the full G-3 aircraft is depicted in Fig. 11.

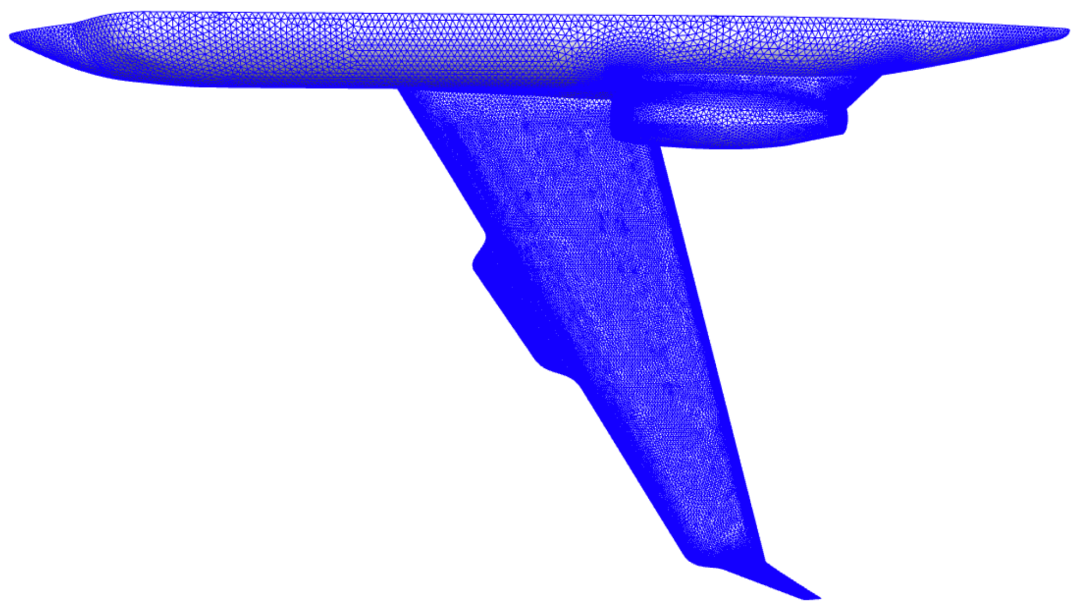

Figure 11. Unstructured surface grid for the G3 aircraft

The grid spacing is determined by Eq. (1) with $r_{1}=0.03$ and $r_{2}=0.005$ in the glove region to ensure a good resolution of approximately 50 points in the boundary layer and $r_{1}=0.12$ and $r_{2}=0.02$ elsewhere to reduce the total grid size. $\Delta_{1}=1.4 e^{-4}$ is used everywhere as in subsection C. The surface grid over the 
gloved wing is shown on the left of Fig. 12. The variable stretching ratios, however, leads to grid skewness in the region with mesh spacing transition and grid discontinuity beyond the boundary-layer edge on the glove, as shown in Fig. 13 for a midspan slice. The grid-skewness also prevents further refinements of the boundary layer mesh. When mean flows are extracted from the FUN3D solutions, the extent of the stability grid in the wall normal direction is always shorter than the distance from the wall where grid discontinuity occurs as shown in Fig. 14.

For validating the mean flow extracted from the FUN3D solution, OVERFLOW is used to perform computations on a structured overset grid, with the gloved wing, fuselage and engine each residing in its own single block. There are around 100-120 points across the boundary layer over the glove region, excluding the leading edge where there are about 30-40 points within the boundary layer. The background Cartesian grid is generated by OVERFLOW automatically. The total number of points in the overset grid is 35.1 million. The right side of Fig. 12 shows the overset grid on the surface of the gloved wing. Fig. 15 displays the overset grids before and after hole cutting at the midspan of the G-3 glove.
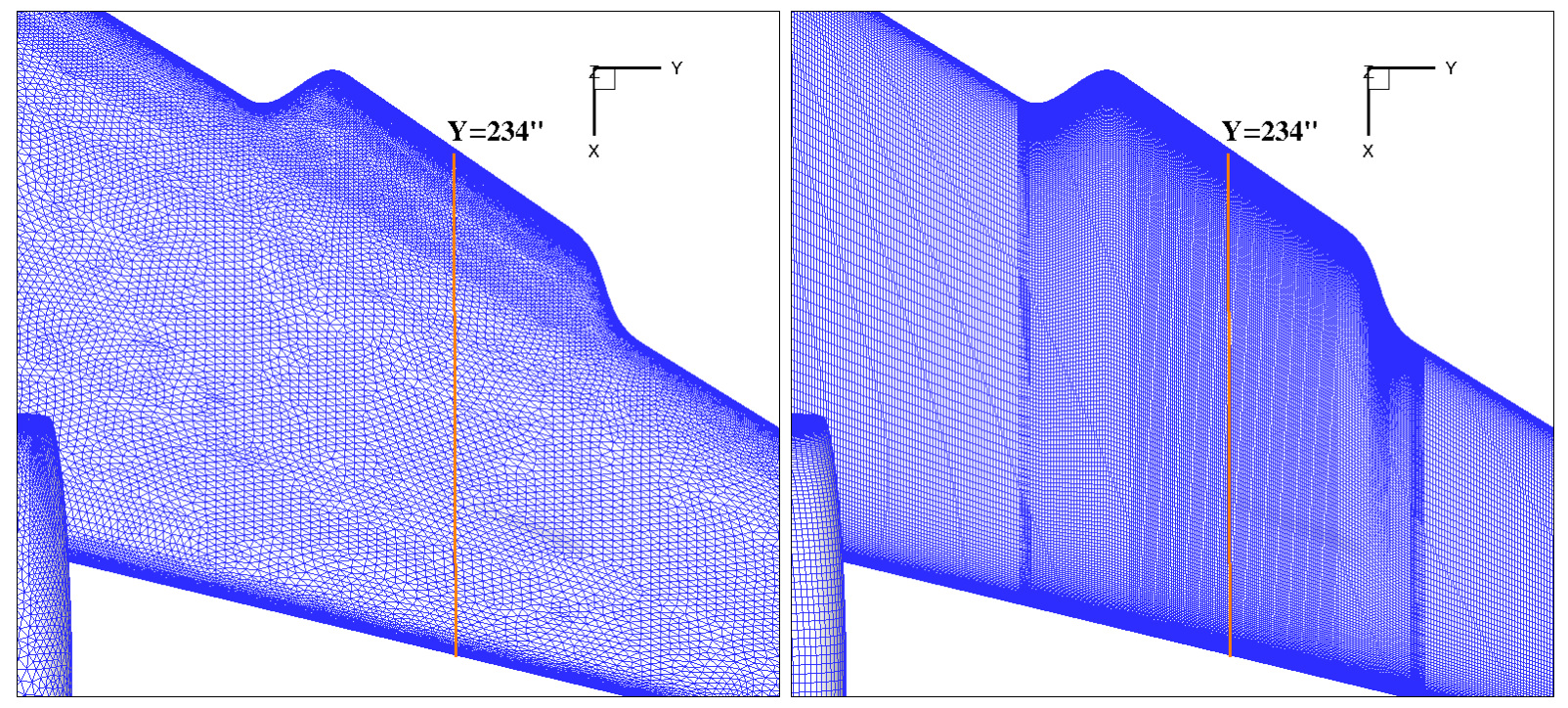

Figure 12. Unstructured (left) and structured (right) grids on the surface of the G-3 aircraft used for the FUN3D and OVERFLOW computations, respectively.

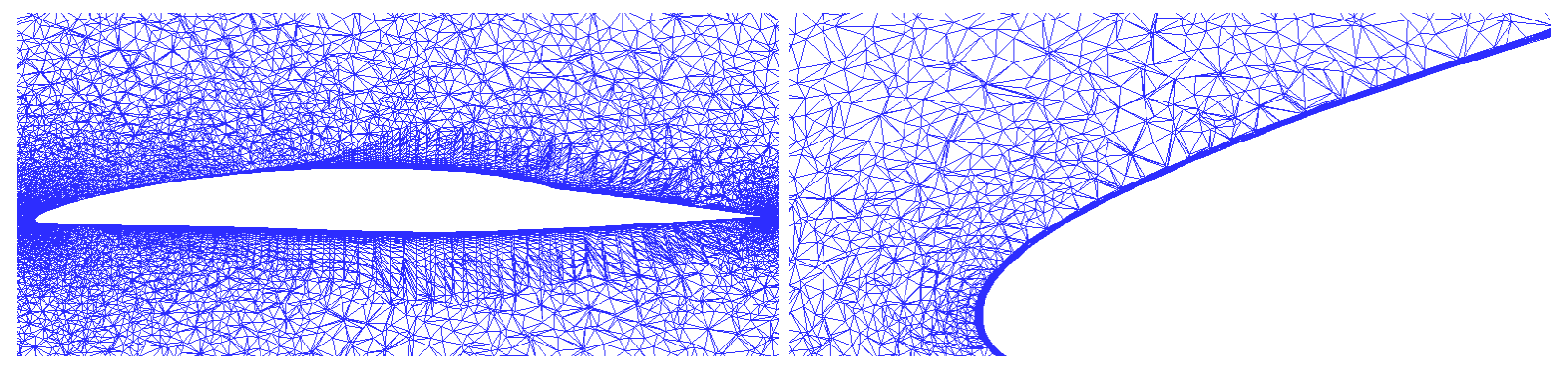

Figure 13. Unstructured grid on the plane at the midspan of the G-3 glove.

The distribution of the pressure (i.e., its gradient) is the most important factor dominating the crossflow development in 3D boundary layer flows. Fig. 16 illustrates the pressure coefficient contours computed by FUN3D and OVERFLOW over the gloved wing of the G-3 aircraft. Good agreement is seen between these two solutions. A streamwise cut is made at the mid-glove location of each solution as shown by the orange lines in Fig. 16 and the pressure coefficient distributions along these cuts are plotted in Fig. 17 with excellent agreements observed between FUN3D and OVERFLOW.

The Crossflow Reynolds number, $R_{C F}$, is an important indicator of the strength of crossflow instability and is, therefore, most relevant for the purpose of validating the extraction procedure designed to construct mean flows for stability analysis. The crossflow Reynolds numbers based on the two solutions along the same 


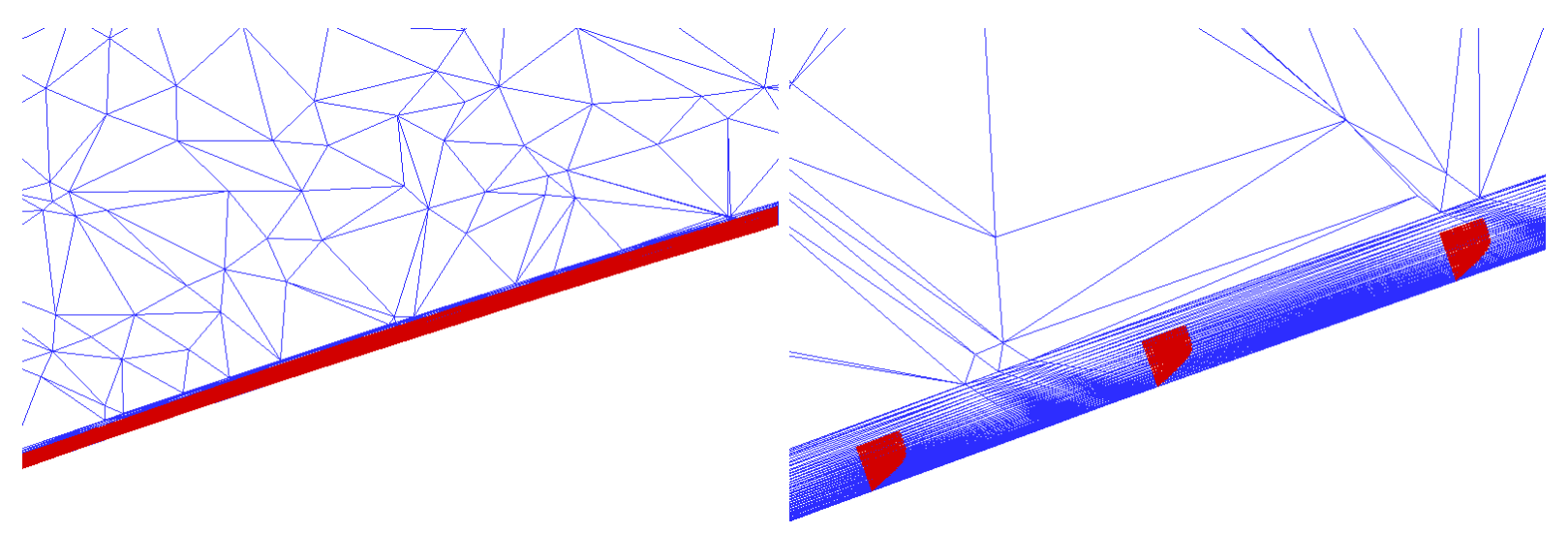

Figure 14. Extent of stability grid in the wall normal direction (left) and extracted mean flow profiles (right) on the plane at the midspan of the G-3 glove.
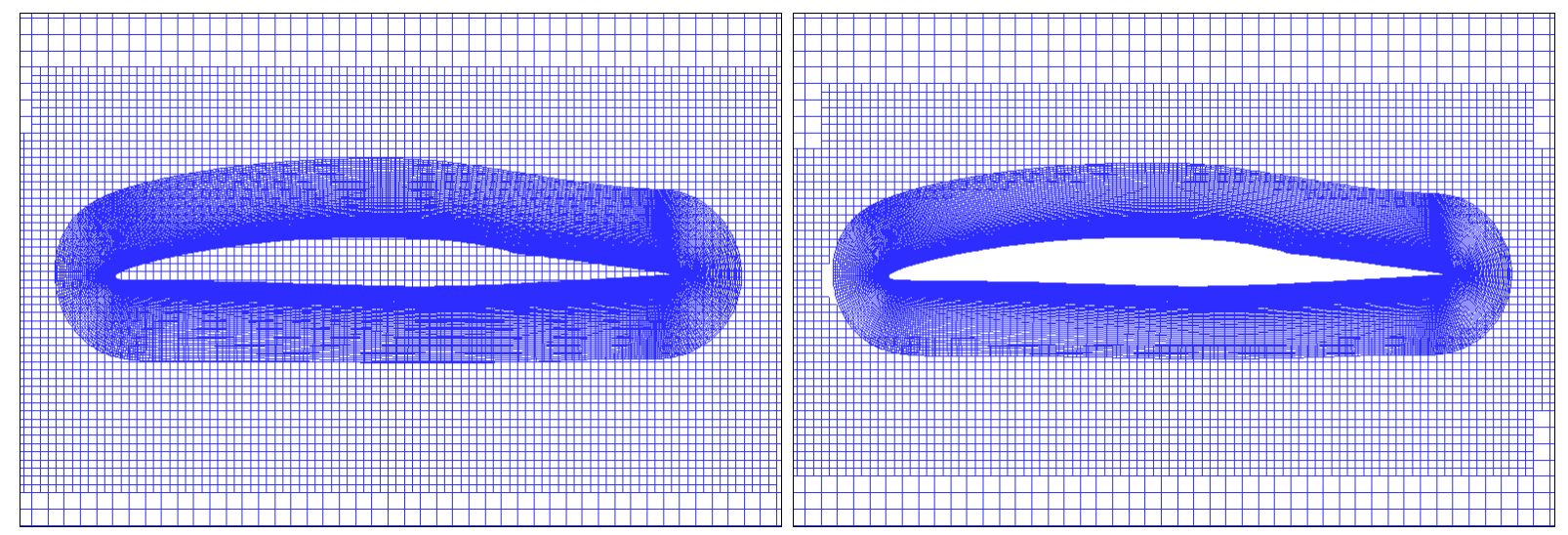

Figure 15. Overset grids before (left) and after (right) hole cutting used for OVERFLOW computations at the midspan of the G-3 glove.

mid-span cut are compared in the upper-left corner of Fig. 18. Good agreement is observed upstream of the chordwise location $x / c=0.34$, beyond which a graphically significant difference begins to appear. Also, nonsmoothness can be seen in $R_{C F}$ for FUN3D. In contrast, there is an excellent agreement between the streamwise Reynolds numbers $R e_{y}$. Note that the mean flow quantities for FUN3D are obtained through interpolation in the extraction procedure while those for OVERFLOW are obtained directly from the values at grid points without any interpolation. When the structured grid is generated for OVERFLOW, it is requested to have a grid plane at the midspan of the glove. In fact, $R_{C F}$ is a much more sensitive quantity than $R e_{y}$ since $R_{C F}$ is related to $U_{C F_{\max }}$ and $\delta_{01}$, both of which are very small quantities. Fig. 18 shows that the magnitude of $U_{C F_{\max }}$ is around 2-3\% of $U_{e} . \delta_{01}$ is the distance from the wall where $U_{C F}$ reduces to $0.1\left|U_{C F_{\max }}\right|$ which implies that $\delta_{01}$ is associated with a quantity even one order smaller than $U_{C F_{\max }}$. From the plots for $\delta_{01}$ and $U_{C F_{\max }}$, one can see similar discrepancy between FUN3D and OVERFLOW as well as nonsmoothness in the FUN3D profiles. Given that the crossflow Reynolds number is such a sensitive quantity and its computation based on Navier-Stokes solutions is subject to uncertainties such as interpolation and determination of the boundary-layer edge, this result is not surprising. The exact reasons behind the discrepancy and nonsmoothness will be further investigated.

Also it is worthy to mention that in the OVERFLOW computations, crossflow component in the boundary layer takes very long time to converge. At time step 6900 with the residual dropped more than 6 orders, the crossflow distribution was not correct and unphysical reversed crossflow was observed while an excellent agreement of $C_{p}$ between OVERFLOW and FUN3D had already been achieved as shown in Fig. 17. The mean flow parameters presented here are obtained at time step 26,000 when the crossflow in the boundary layer has achieved full convergence. 


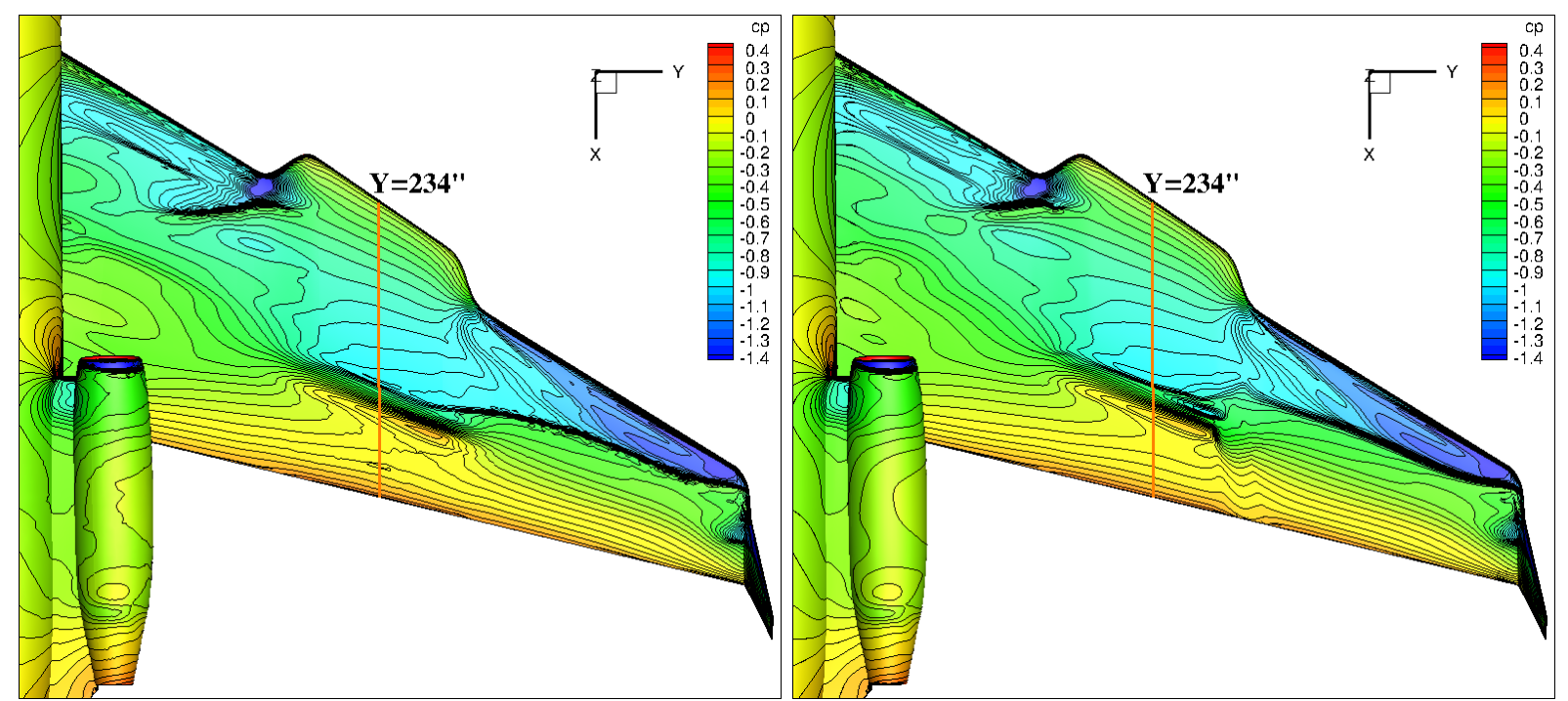

Figure 16. $C_{p}$ contours computed by FUN3D (left) and OVERFLOW (right) on the surface of G-3 with the gloved wing.

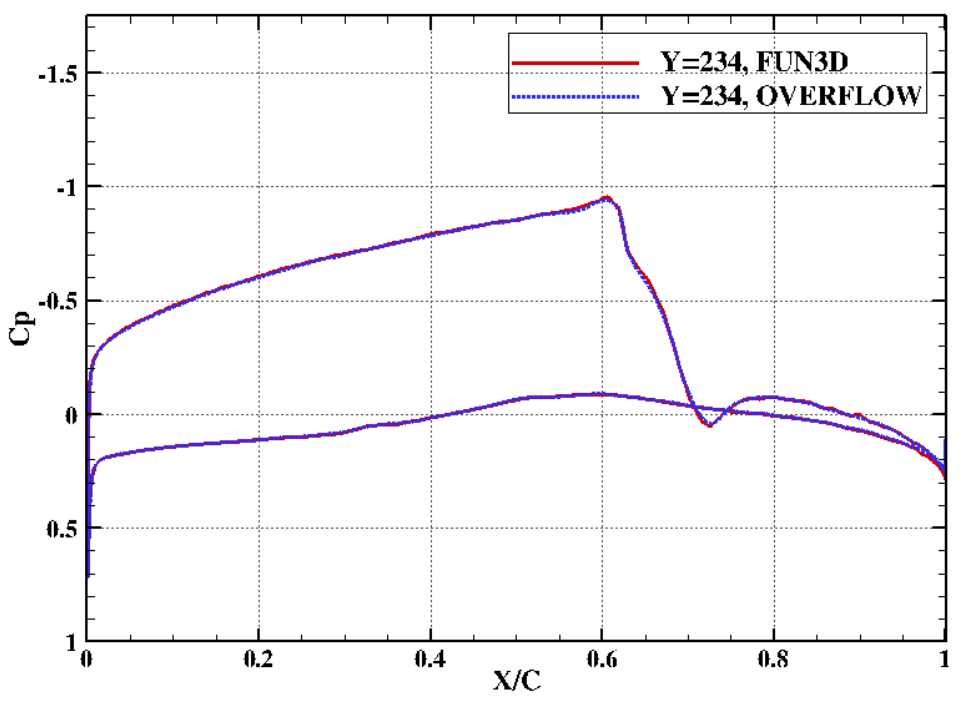

Figure 17. Comparison of the $C_{p}$ distributions calculated by FUN3D and OVERFLOW on the midspan of the glove.

As in subsection $\mathrm{C}$, three-dimensional linear stability analysis is performed here on each of the mean flows for stationary crossflow instability modes with spanwise wavelengths of 5, 7,9 and $11 \mathrm{~mm}$, respectively, along a streamline in mid-glove region. The comparison of $\mathrm{N}$-factors is shown in Fig. 19 with generally good agreement for small values of $x / c$. However, small differences are observed in downstream regions, which could be attributed to the difference observed in $R_{C F}$ shown in Fig. 18. The reasons for the discrepancy are subject to further examination in our future work.

Boundary-layer codes are widely used for generating mean flows for stability analysis for 2D as well as 3D wings because of their efficiency and simplicity. Therefore, the stability analysis results based, respectively, on mean flows generated by a boundary layer solver and a Navier-Stokes solver are evaluated and compared. The boundary-layer code BLSTA is used for this purpose because it can handle not only infinite swept wings, but also tapered swept wings under a spanwise conical flow assumption. $C_{p}$ along the mid-span and on the upper surface of the glove is used as the boundary-layer edge condition for BLSTA. Three mean flows are generated: (1) infinite swept wing by BLSTA; (2) tapered swept wing by BLSTA with the trailing edge 

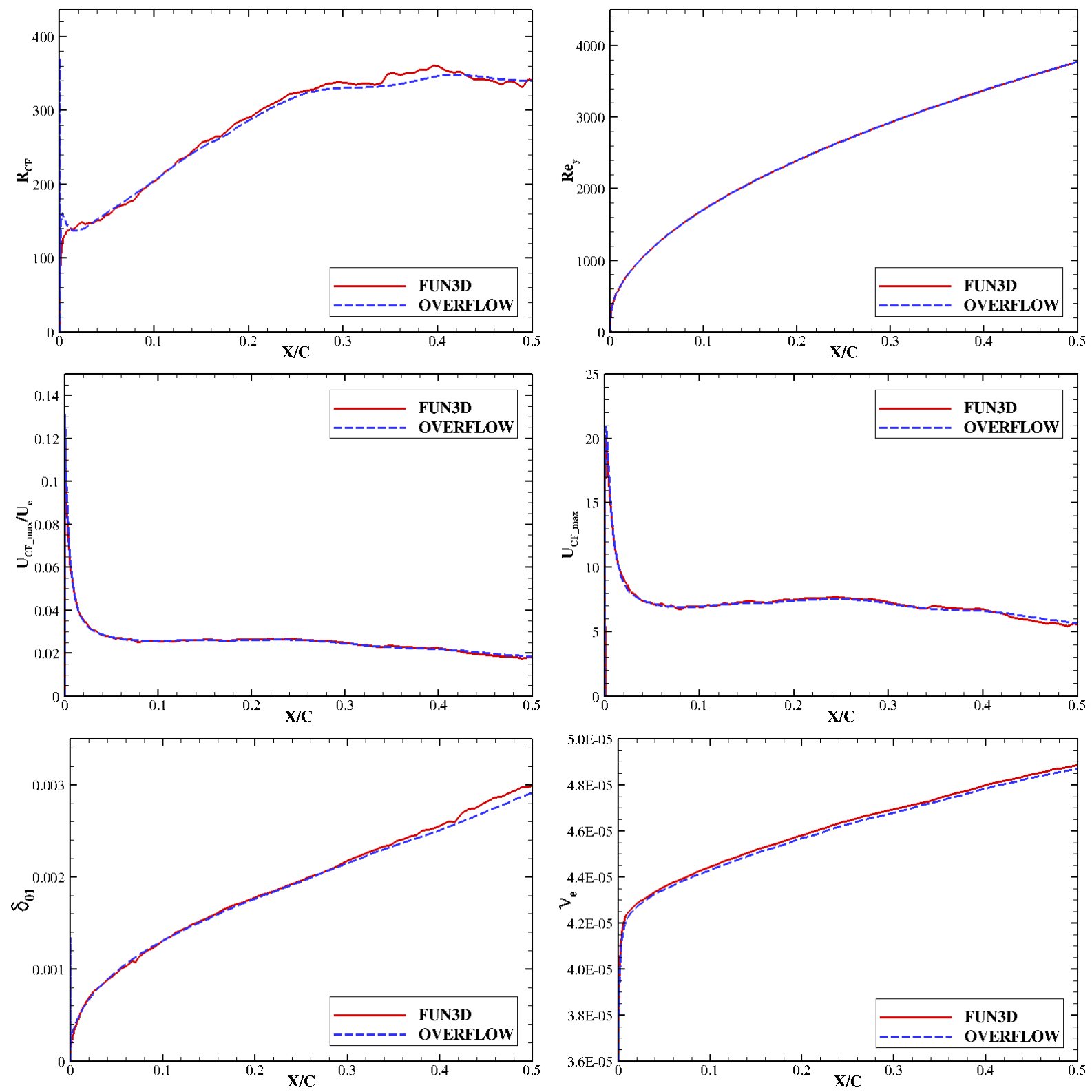

Figure 18. Comparison of various mean flow parameters, including the streamwise Reynolds number $\left(R e_{y}\right)$, the crossflow Reynolds number $\left(R_{C F}\right)$, the dimensional (in $\mathrm{m} / \mathrm{s}$ ) and non-dimensional maximum crossflow velocity $\left(U_{C F_{\max }}\right.$ and $\left.\frac{U_{C F_{\max }}}{U_{e}}\right)$, the crossflow length scale $\left(\delta_{01}\right.$, in $m$ ), and the boundary layer edge kinematic viscosity $\left(\nu_{e}\right.$, in $\left.\mathrm{m}^{2} / \mathrm{s}\right)$, based on FUN3D and OVERFLOW solutions.

sweep angle set to $13.62^{\circ}$; (3) the mean flow extracted from the FUN3D Navier-Stokes solutions. Stability computations are performed for stationary crossflow instability modes of 3 to $18 \mathrm{~mm}$ in wavelength, with the 3D integration path being a streamline in case (3). The resulting N-factors are shown in Fig. 20. The $\mathrm{N}$-factors of case (3) are only $42 \%-57 \%$ of those of case (1) depending on spanwise wavelength. In case (2), the N-factors drop 10\% - $15 \%$ from those in case (1) while they are still significantly larger than those in case (3).

The pressure distribution dominates the development of crossflow on a swept wing. We will, therefore, examine the $C_{p}$ contours over the glove to find possible reasons for such big difference between $2 \mathrm{D}$ and $3 \mathrm{D}$ stability analysis. In Fig. 21 , we illustrated the local sweep lines at $x / c=0.3,0.4$, and 0.6 together with $C_{p}$ contours in the glove area between two span stations, $y=204$ " and $y=264$ ". It is seen that the local $C_{p}$ contours are not parallel to either the leading edge or the local sweep lines. For instance, 

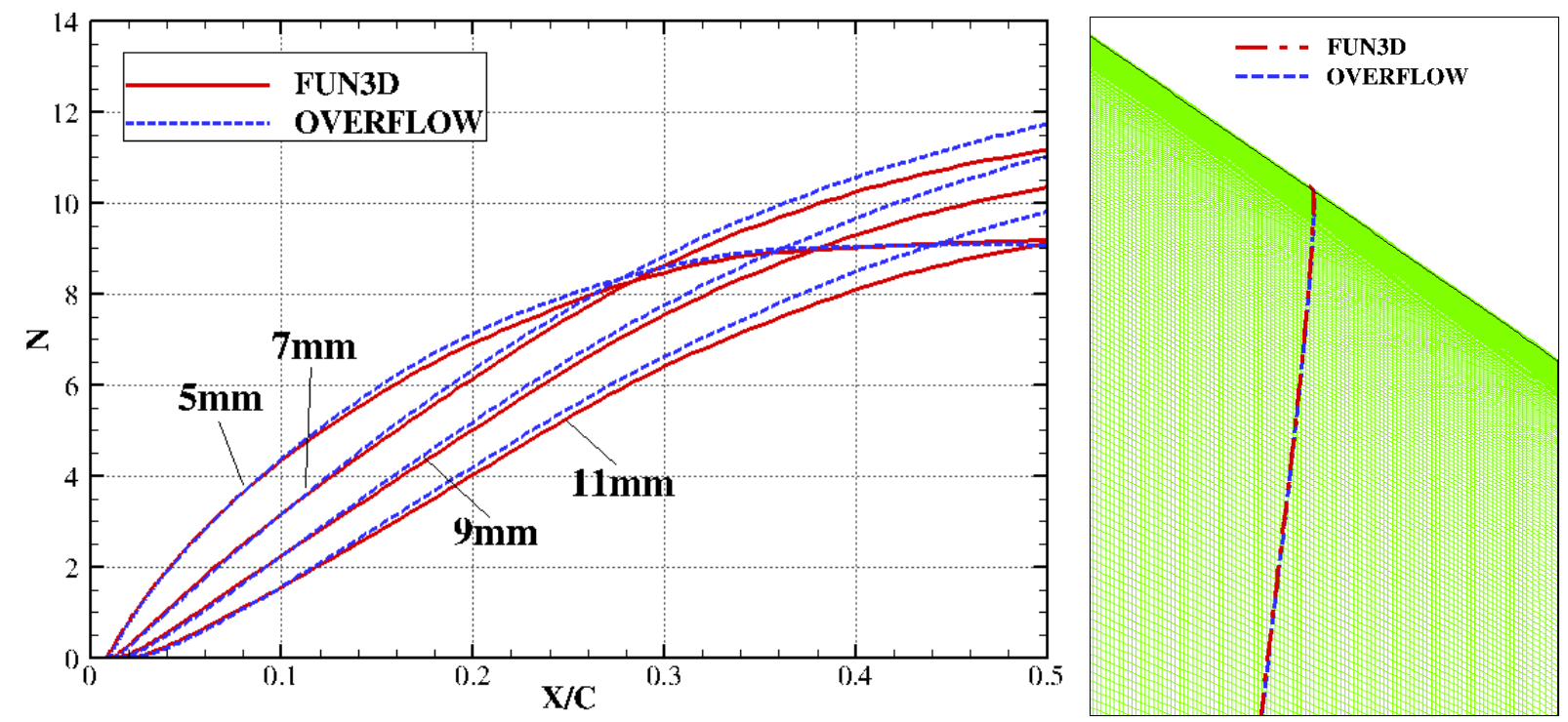

Figure 19. 3D LST N-factors for four stationary crossflow modes, 5, 7, 9, and $11 \mathrm{~mm}$, based on the mean flow profiles from full G-3 computations conducted by FUN3D and OVERFLOW (left) and the corresponding streamlines along which the stability analysis was conducted (right).

between $x / c=0.4$ and 0.6 , the actual sweep angles defined by the local $C_{p}$ contours approach zero which is much less than the sweep angle defined by the local sweep lines. It appears that the separation zone at the leading edge of the inboard fairing as well as the flow near the outboard fairing play an important role in the change of the downstream $C_{p}$ distributions. The isobar un-sweep in the glove region reduces crossflow, which weakens crossflow instability. A larger glove span, or perhaps a more clever design of side fairings, would have minimized this stabilizing effect. In any case, quasi-3D boundary-layer codes (such as BLSTA) are not suitable for computing the fully three-dimensional glove boundary layer. Navier-Stokes codes, as in the present paper, or much more efficient fully $3 \mathrm{D}$ boundary layer $\operatorname{codes}^{29}$ are needed to compute the mean flow for stability analysis.

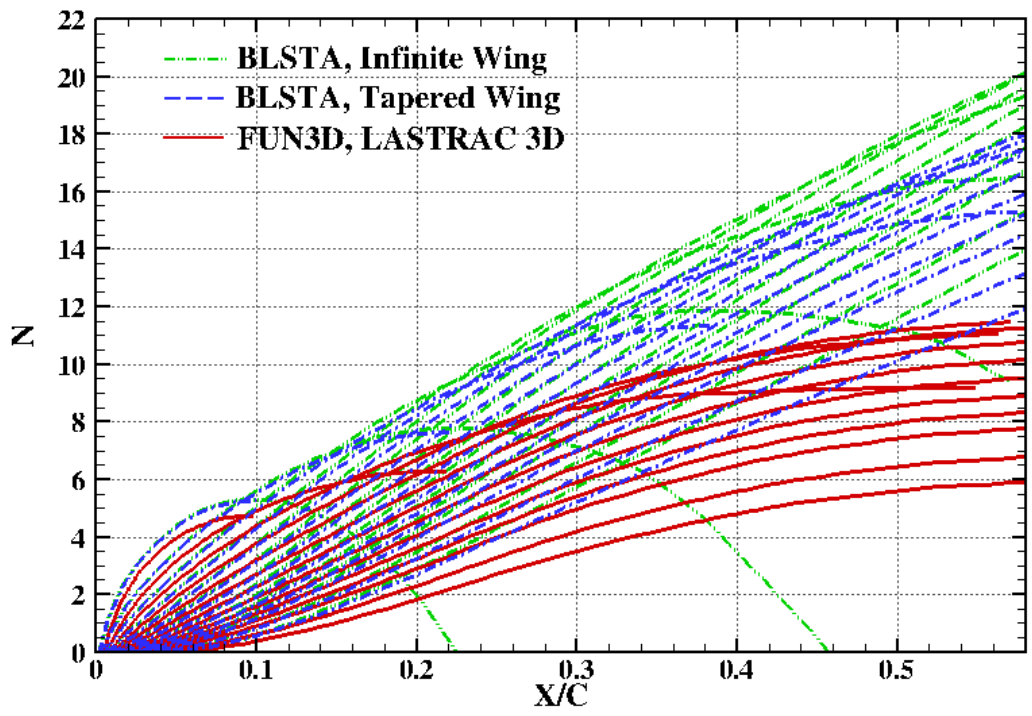

Figure 20. Comparison of 2D (based on quasi-3D boundary layer mean flows) and 3D (based on Navier-Stokes mean flows) LST N-factors for stationary crossflow wavelengths from $3 \mathbf{m m}$ to $18 \mathrm{~mm}$ based on the mean flows obtained by the boundary layer code, BLSTA, and by the direct extraction from the FUN3D solutions. 


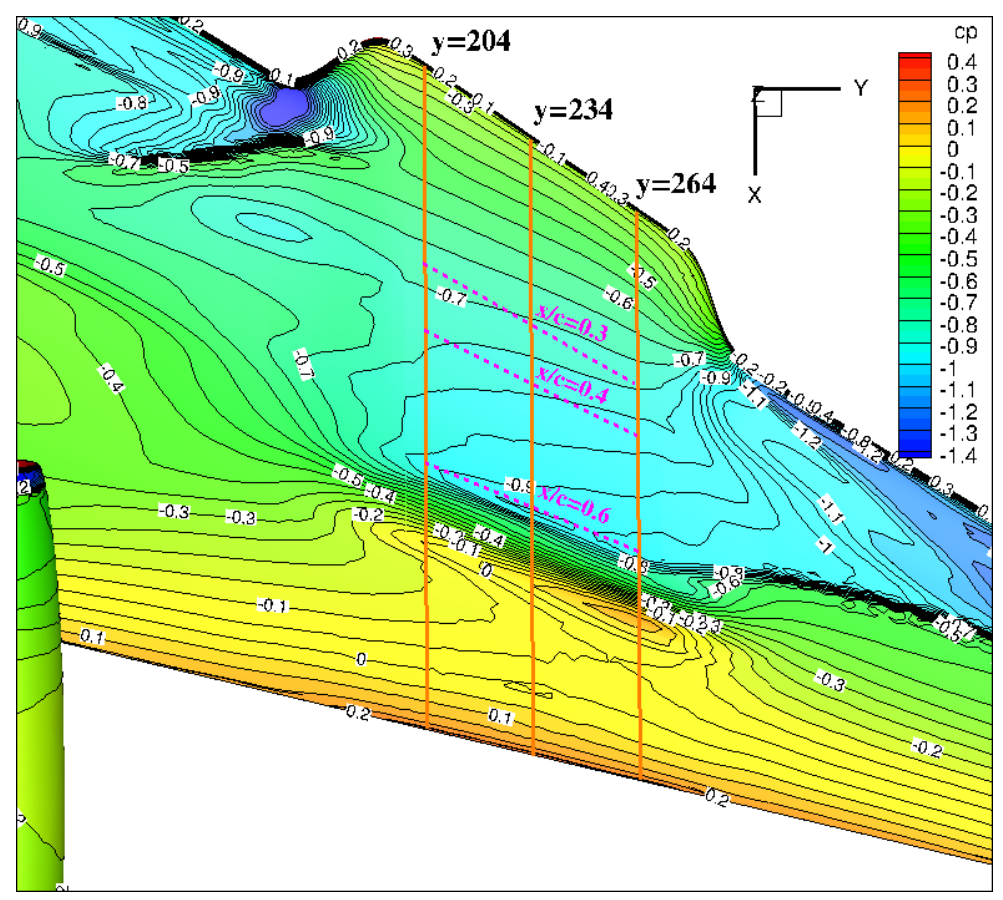

Figure 21. Cp contours over the glove for the full G3 aircraft together local sweep lines.

\section{Summary and Concluding Remarks}

The work described in this paper is motivated by the longer term goal of developing efficient 3D design capability for swept wings with laminar flow technology. Because of the large number of design variables inherent to such applications, an adjoint-based design optimization capability becomes highly desirable in this context. The unstructured grid flow solver FUN3D, developed at NASA Langley, includes a number of essential ingredients for the targeted capability in the form of adjoint based optimization, built-in error estimation, and objective based adaptive mesh refinement. However, additional effort is necessary to enable linear stability based transition predictions for fully 3D mean flows using unstructured grid flow solutions.

Since the 3D transition analysis module within the LASTRAC suite of transition analysis codes is based on boundary layer profiles at each point of a structured grid surface mesh, a post-processing module is developed and implemented to interface the unstructured grid mean flow solution from FUN3D with the LASTRAC suite of codes. The above procedure has been applied to a variety of relevant flow configurations ranging in complexity from a self-similar flat plate boundary layer, an infinite-span swept wing, a 3D wing configuration, to a full aircraft configuration with a gloved wing. In all cases, the computed mean flow solutions as well as the corresponding linear stability characteristics are compared with the predictions based on more established methods from analytical solution to solutions using structured grid mean flow solvers. Good agreements are observed among the results obtained by these methods. Overall, the study provides useful guidelines for future FUN3D applications for design and analysis of complex flow configurations related to laminar flow technology.

For the swept wing flow over the wing-glove assembly, it is also demonstrated that the $\mathrm{N}$-factor evolution based on a full-Navier-Stokes computation differs significantly from that based on quasi-3D boundary layer codes owing to the un-sweep of the isobars caused by the limited glove span. This points to the need for stability analysis based on Navier-Stokes solutions or possibly fully 3D boundary layer codes when the underlying flow develops strong three-dimensionality.

\section{Acknowledgments}

The current work was supported by NASA's Environmentally Responsible Aviation and Subsonic Fixed Wing projects. The authors thank Dr. Robert Biedron, Dr. Christopher Rumsey, and Dana Hammond of 
NASA Langley Research Center for their valuable suggestions and fruitful discussions.

\section{References}

${ }^{1}$ Chang, C.-L., "The Langley Stability and Transition Analysis Code (LASTRAC): LST, Linear and Nonlinear PSE for 2-D, Axisymmetric, and Infinite Swept Wing Boundary Layers," AIAA Paper 2003-0974, 2003.

${ }^{2}$ Chang, C.-L., "LASTRAC.3d: Transition in 3D Boundary Layers," AIAA Paper 2004-2542, 2004.

${ }^{3}$ Anderson, W. K. and Bonhaus, D. L., "An Implicit Upwind Algorithm for Computing Turbulent Flows on Unstructured Grids," Comput. Fluids, Vol. 23, 1994, pp. 1-22.

${ }^{4}$ Anderson, W. K., Rausch, R. D., and Bonhaus, D. L., "Implicit/Multigrid Algorithms for Incompressible Turbulent Flows on Unstructured Grids," J. Comput. Phys., Vol. 128, 1996, pp. 391-408.

${ }^{5}$ Nielsen, E. J., "Aerodynamic Design Sensitivities on an Unstructured Mesh Using the Navier-Stokes Equations and a Discrete Adjoint Formulation," Ph.D thesis, Virginia Polytechnic Institute and State University, Blacksburg, VA, 1998.

${ }^{6}$ Lee-Rausch, E. M., Park, M. A., Jones, W. T., Hammond, D. P., and Nielsen, E. J., "Application of Parallel Adjoint-Based Error Estimation and Anisotropic Grid Adaptation for Three-Dimensional Aerospace Configurations," AIAA Paper 2005-4842, 2005. 2011.

${ }^{7}$ Park, M., "Low Boom Configuration Analysis with FUN3D Adjoint Simulation Framework," AIAA Paper 2011-3337,

${ }^{8}$ Nielsen, E. J. and Diskin, B., "Discrete Adjoint-Based Design for Unsteady Turbulent Flows on Dynamic Overset Unstructured Grids," AIAA Paper 2012-0554, 2012.

${ }^{9}$ Belisle, M. J., Roberts, M. W., Tufts, M. W., Tucker, A. A., Williams, T. C., Saric, W. S., and Reed, H. L., "Design of the Subsonic Aircraft Roughness Glove Experiment (SARGE)," AIAA Paper 2011-3524, 2011.

${ }^{10}$ Malik, M., Liao, W., Lee-Rausch, E., Li, F., Choudhari, M., and Chang, C.-L., "Computational Analysis of the G-III Laminar Flow Glove," AIAA Paper 2011-3525, 2011.

${ }^{11}$ Hartshorn, F., Belisle, M. J., and Reed, H. L., "Computational Optimization of a Natural Laminar Flow Experimental Wing Glove," AIAA Paper 2012-0870, 2008.

${ }^{12}$ Krist, S. L., Biedron, R. T., and Rumsey, C. L., "CFL3D User's Mannual (Version 5.0)," NASA-TM-1998-208444, 1998.

${ }^{13}$ Rumsey, C. L. and Lee-Rausch, E. M., "NASA Trapezoidal Wing Computations Including Transition and Advanced Turbulence Modeling," 42nd AIAA Fluid Dynamics Conference and Exhibit, New Orleans, LA, 2012.

${ }^{14}$ Nichols, R. and Buning, P., User's Manual for OVERFLOW 2.1, NASA Langley Research Center, Hampton, VA, 2008.

${ }^{15}$ Nichols, R., R., T., and Buning, P., "Solver and Turbulence Model Upgrades to OVERFLOW 2 for Unsteady and High-Speed Applications," AIAA Paper 2006-2824, 2006.

${ }^{16}$ Ramakrishnan, R., Vatsa, V., Otto, J., and Kumar, A., "A Detailcd Study of Mean-Flow Solutions for Stability Analysis of Transitional Flows," AIAA Paper 1993-3052, 1993.

${ }^{17}$ Garriz, J., Vatsa, V., and Sanetrik, M., "Issues Involved in Coupling Navier-Stokes Mean-Flow and Linear Stability Codes," AIAA Paper 1994-0304, 1994.

${ }^{18}$ Malik, M., Li, F., and Choudhari, M., "Analysis of Crossflow Transition Flight Experiment aboard Pegasus Launch Vehicle," AIAA Paper 2007-4487, 2007.

${ }^{19}$ Roe, P. L., "Approximate Riemann Solvers, Parameter Vectors, and Difference Schemes," J. Comput. Phys., Vol. 43, 1981, pp. 357-372.

${ }^{20}$ Pirzadeh, S., "Progress Toward a User-Oriented Unstructured Viscous Grid Generator," AIAA Paper 1996-0031, 1996.

${ }^{21}$ Jameson, A., Schmidt, W., and Turkel, E., "Numerical Solutions of the Euler Equations by Finite Volume Methods Using Runge-Kutta Time-Stepping Schemes," AIAA Paper 1981-1259, 1981.

${ }^{22}$ Malik, M., " $e^{\text {Malik }}:$ A New Spatial Stability Analysis Program for Transition Prediction Using the $e^{N}$ Method," HTC Report No. HTC-8902, 1989.

${ }^{23}$ Pruett, D. C. and Streett, C. L., "A Spectrally Accurate Boundary-Layer Code for Infinite Swept Wings," NASA CR195014, 1994.

${ }^{24}$ Wie, Y. S., "BLSTA-A Boundary Layer Code for Stability Analysis," NASA CR-4481, 1992.

${ }^{25}$ Schlichting, H. and Gersten, K., Boundary Layer Theory, Springer, 2004.

${ }^{26}$ Lee-Rausch, E. M., Frink, N. T., Mavriplis, D. J., Rausch, R. D., and Milholen, R. D., "Transonic Drag Prediction on a DLR-F6 Transport Configuration Using Unstructured Grid Solvers," AIAA Paper 2004-0554, 2004.

${ }^{27}$ Spalart, P. R. and Allmaras, S. R., "A One-EquationTurbulence Model for Aerodynamic Flows," La Recherche Aerospatiale, Vol. 1, 1994, pp. 5-21.

${ }^{28}$ Fletcher, H., Dryden Flight Research Center, Private Communication, 2011.

${ }^{29}$ Iyer, V., "Three-Dimensional Boundary-Layer Program (BL3D) for Swept Subsonic or Supersonic Wings with Application to Laminar Flow Control," NASA CR-4531, 1989. 